\title{
COMPLEX SCALING AND THE DISTRIBUTION OF SCATTERING POLES
}

\author{
JOHANNES SJÖSTRAND AND MACIEJ ZWORSKI
}

\section{INTRODUCTION AND STATEMENT OF RESULTS}

The purpose of this paper is to establish sharp polynomial bounds on the number of scattering poles for a general class of compactly supported self-adjoint perturbations of the Laplacian in $\mathbb{R}^{n}, n$ odd. We also consider more general types of bounds that give sharper estimates in certain situations. The general conclusion can be stated as follows: The order of growth of the poles is the same as the order of growth of eigenvalues for corresponding compact problems. From the few known cases, however, the exact asymptotics are expected to be different.

The scattering poles for compactly supported perturbations were rigorously defined by Lax and Phillips [13]. In a more general setting they correspond to resonances, the study of which has a long tradition in mathematical physics. In the Lax-Phillips theory they appear as the poles of the meromorphic continuation of the scattering matrix and coincide with the poles of the meromorphic continuation of the resolvent of the perturbed operator. Because of the latter characterization, they can be considered as the analogue of the discrete spectral data for problems on noncompact domains.

The problem of estimating the counting function

$$
N(r)=\#\left\{\lambda_{j}:\left|\lambda_{j}\right| \leq r, \lambda_{j} \text { is a scattering pole }\right\},
$$

where the poles are included according to their multiplicities, was introduced by Melrose [17], who after obtaining a polynomial bound for a general class of problems [18, 19] established a sharp bound

$$
N(r)=\mathscr{O}\left(r^{n}\right)
$$

in the case of obstacle scattering [20]. The bound (1.2) was then obtained in the case of scattering by compactly supported bounded potentials by the second author [29] (the exponent $n+1$ was obtained earlier by Melrose). Vodev [26] has recently extended that result to compactly supported metric perturbations.

Received by the editors February 25, 1991.

1991 Mathematics Subject Classification. Primary 35P25, 47A40.

The second author's research was partially supported by NSF Grant 8922720 .

(C) 1991 American Mathematical Society $0894-0347 / 91 \$ 1.00+\$ .25$ per page 
As one would expect, more detailed results are possible in one dimension and in the radial case. It is shown in [27] that for potentials $V \in L_{\text {comp }}^{1}(\mathbb{R})$

$$
N(r)=\frac{2}{\pi} \times \sup _{x, y \in \operatorname{supp} V}|x-y| r+o(r),
$$

while for sufficiently regular radial potentials in $\mathbb{R}^{n}, n$ odd, nonvanishing at the boundary of the support [28],

$$
N(r)=C_{n}\left(\sup _{x \in \operatorname{supp} V}|x|\right)^{n} r^{n}+o\left(r^{n}\right) .
$$

For compactly supported vector potential perturbations, Intissar [11] gave a bound with the exponent $n+1$. He was also able to obtain some bounds in the even-dimensional case [12].

The resonances, which constitute a more general class of objects, have been studied, in the semiclassical setting by the method of complex scaling that was rigorously introduced by Aguilar-Combes [1] and Balslev-Combes [2], then extended by several authors, see for instance Hunziker [10] and Helffer-Martinez [5].

A microlocal approach to complex scaling was developed by Helffer and the first author [6]. The density of the resonances for small Planck constant was then studied by the first author in [23] (announced in [24]) and was related to the dynamical properties of the operator. Under the general assumption of [6], it was also shown there that the number of resonances, $N(h)$, in a small $h$-independent neighbourhood of 0 satisfies

$$
N(h)=\mathscr{O}\left(h^{-n}\right) \text {, }
$$

which is the semiclassical analogue of (1.2). Specialized to the case of compactly supported perturbations of Laplacian, the same approach yields the bound (1.2) for poles in a conic neighbourhood of the real axis, and that was our starting point-we extend some of the methods of [23] to estimate the density of the poles in discs.

We make the following general assumptions on the operator $P$, a compactly supported perturbation of $-\Delta$.

Let $\mathscr{H}$ be a complex Hilbert space with an orthogonal decomposition,

$$
\mathscr{H}=\mathscr{H}_{R_{0}} \oplus L^{2}\left(\mathbb{R}^{n} \backslash B\left(0, R_{0}\right)\right),
$$

where $R_{0}>0$ is some fixed constant and $B(x, R)=\left\{y \in \mathbb{R}^{n}:|x-y|<R\right\}$. The corresponding orthogonal projections will be denoted $\left.u \mapsto u\right|_{B\left(0, R_{0}\right)}$ and $\left.u \mapsto u\right|_{\mathbb{R}^{n} \backslash B\left(0, R_{0}\right)}$. The operator

$$
P: \mathscr{H} \rightarrow \mathscr{H}
$$

is assumed to be unbounded, selfadjoint with domain $\mathscr{D} \subset \mathscr{H}$. We also assume that

$$
\left.\mathscr{D}\right|_{\mathbb{R}^{n} \backslash B\left(0, R_{0}\right)} \subset H^{2}\left(\mathbb{R}^{n} \backslash B\left(0, R_{0}\right)\right),
$$


and conversely, $u \in \mathscr{D}$ if $u \in H^{2}\left(\mathbb{R}^{n} \backslash B\left(0, R_{0}\right)\right)$ and $u$ vanishes near $B\left(0, R_{0}\right)$;

$$
\begin{gathered}
\left.(P u)\right|_{\mathbb{R}^{n} \backslash B\left(0, R_{0}\right)}=-\Delta\left(\left.u\right|_{\mathbb{R}^{n} \backslash B\left(0, R_{0}\right)}\right) \text { for all } u \in \mathscr{D} ; \\
\mathbb{1}_{B\left(0, R_{0}\right)}(P+i)^{-1} \text { is compact. }
\end{gathered}
$$

We also define $\mathscr{H}_{\text {comp }}=\mathscr{H}_{R_{0}} \oplus L_{\text {comp }}^{2}\left(\mathbb{R}^{n} \backslash B\left(0, R_{0}\right)\right)$ and $\mathscr{H}_{\text {loc }}$ similarly. Analogously,

$$
\mathscr{D}_{\text {loc }}=\left\{u \in \mathscr{H}_{\text {loc }}: \chi u \in \mathscr{D} \text { for } \chi \in C_{0}^{\infty}\left(\mathbb{R}^{n}\right) \text {, constant near } B\left(0, R_{0}\right)\right\} .
$$

The results in $\S 3$ give the following

Theorem 1.1. Under the assumptions on $P$ above and for $n$ odd, the resolvent

$$
\left(P-\lambda^{2}\right)^{-1}: \mathscr{H} \rightarrow \mathscr{D}, \quad \Im \lambda>0, \lambda^{2} \notin \sigma_{\text {point }}(P),
$$

has a meromorphic continuation to $\mathbb{C}$ :

$$
\left(P-\lambda^{2}\right)^{-1}: \mathscr{H}_{\text {comp }} \rightarrow \mathscr{D}_{\text {loc }} .
$$

The scattering poles are defined as the poles of $\left(P-\lambda^{2}\right)^{-1}$. The multiplicity of a pole $\lambda_{0}$ is given in terms of multiplicity of the corresponding resonance $z_{0}=\lambda_{0}^{2}$, multiplicity of $\lambda_{0}=\operatorname{dim} \pi_{\lambda_{0}^{2}}\left(\mathscr{H}_{\text {comp }}\right)$,

$$
\pi_{z_{0}}=(2 \pi i)^{-1} \int_{\gamma}(z-P)^{-1} d z: \mathscr{H}_{\text {comp }} \rightarrow \mathscr{D}_{\text {loc }} \gamma:\left[0,2 \pi\left[\ni \sigma \mapsto z_{0}+\varepsilon e^{i \sigma} .\right.\right.
$$

To estimate (1.1) we need the following spectral assumption: Let $P_{0}=h^{2} P$, $h \in] 0,1]$, and let $g \in C_{0}^{\infty}(\mathbb{R})$ and $\chi \in C_{0}^{\infty}\left(\mathbb{R}^{n}\right)$, constant near $B\left(0, R_{0}\right)$. Then

$$
\left|\operatorname{tr}\left(g\left(P_{0}\right) \chi\right)\right| \leq C_{g} \Phi\left(C_{g} h^{-1}\right),
$$

where $\Phi:[1, \infty) \rightarrow[1, \infty)$ is an increasing function such that $\Phi(t) \geq t^{n}$ and such that there exist $\widetilde{C}, \delta>0$ with the property $\Phi(\theta t) \leq \widetilde{C} \theta^{\delta} \Phi(t)$ for all $\theta, t$ with $0<\theta \leq 1, t \geq 1, \theta t \geq 1$.

As we shall see this is satisfied in many interesting situations. The simplest $\Phi$ to consider is

$$
\Phi(t)=C t^{\tilde{n}}, \quad \tilde{n} \geq n .
$$

Theorem 1.2. Under the general assumptions on $P$ above, and under the assumption (1.7), the counting function for the scattering poles (1.1) satisfies

$$
N(r) \leq C \Phi(C r)
$$

for some constant $C$ and $r \geq 1$.

The assumption (1.7) can be replaced by a bound on the number of eigenvalues for a compactified problem obtained by imposing boundary conditions in a domain containing $B\left(0, R_{0}\right)$, see Remark 7.1.

Theorem 1.2 covers all previously known cases and actually we have 
Corollary 1.1. Let $\mathscr{O}=\mathbb{R}^{n} \backslash K$ be a connected exterior domain, $n$ odd, $K$ compact with a $C^{\infty}$ boundary, $\partial K$. Let $P$ be a symmetric second order operator

$$
P(x, D)=-\sum \frac{\partial}{\partial x_{j}}\left(g^{j k} \frac{\partial}{\partial x_{k}}\right)+\sum b^{j} \frac{\partial}{\partial x_{j}}+c
$$

with coefficients in $\bar{C}^{\infty}(\mathscr{O})$ and such that $(1.5)$ holds, $\left(g^{j k}\right)$ real and positive definite. Then for the operator defined by $P$ on $L^{2}(\mathscr{O})$ with the Dirichlet boundary condition

$$
\left.u\right|_{\partial \mathscr{O}}=0
$$

the number of scattering poles satisfies

$$
N(r)=\mathscr{O}\left(r^{n}\right) \text {. }
$$

For simplicity we only stated the simplest boundary condition above; the same result holds for selfadjoint Robin boundary conditions.

The sharp polynomial bound on the number of scattering poles allows the application of the Tauberian Theorem to obtain asymptotics of the scattering phase. This important observation was made by Melrose [21], who used it to obtain the Weyl law for the scattering phase in the obstacle case. Corollary 1.1 above extends that method to more general scattering problems. Again we shall restrict to the simplest application and consider the operator

$$
-\Delta+V(x) \text { on } \mathscr{O}=\mathbb{R}^{n} \backslash K
$$

with the Dirichlet boundary condition on $\partial K, V \in C_{0}^{\infty}(\overline{\mathscr{O}})$. If $S_{V, K}(\lambda)$ is the scattering matrix for this problem and

$$
s_{V, K}(\lambda)=-i \log \operatorname{det} S_{V, K}(\lambda), \quad \lambda \in \mathbb{R},
$$

is the scattering phase, then Melrose's theorem holds for $s_{V, K}$ as well.

Corollary 1.2. For scattering by a smooth compact obstacle $K \subset \mathbb{R}^{n}, n$ odd, and a potential $V \in C_{0}^{\infty}\left(\overline{\mathbb{R}^{n} \backslash K}\right)$ with Dirichlet boundary conditions,

$$
s_{V, K}(\lambda)=C_{n} \operatorname{vol}(K) \lambda^{n}+O\left(\lambda^{n-1}\right), \quad \lambda \rightarrow \infty .
$$

If the measure of the set of closed transversally reflected geodesics in $T^{*}\left(\mathbb{R}^{n} \backslash K\right)$ is zero then (1.10) can be improved to

$$
s_{V, K}(\lambda)=C_{n} \operatorname{vol}(K) \lambda^{n}+C_{n}^{\prime} \operatorname{vol}(\partial K) \lambda^{n-1}+o\left(\lambda^{n-1}\right) .
$$

Let us finally mention two situations where the exponent $\tilde{n}$ in (1.8) might be greater than $n$.

For that let $P$ be of the form given by (1.9) with real coefficients. Let us assume that $P$ is positive but that it satisfies only subelliptic estimates,

$$
\|u\|_{2 \varepsilon+k}<C\|P u\|_{k}+C\|u\|_{k}, \quad \varepsilon>0 \text {. }
$$

Since condition (1.7) does not change if we add a constant to $P$, we can assume that $P$ is bounded away from 0 so that (1.11) yields

$$
\|u\|_{2 l \varepsilon} \leq C\left\|P^{l} u\right\| \quad(\varepsilon \leq 1) .
$$


As in the case of Corollary 1.1 (see $\S 2)$ this implies that the spectral function satisfies

$$
|e(\lambda, x, x)| \leq C \lambda^{(n+\delta(1-\varepsilon)) / 2 \varepsilon} \text { for all } \delta>0
$$

and (1.7) holds with any

$$
\tilde{n}>n / \varepsilon \text {. }
$$

We can take as an example the operator

$$
P=D_{x_{1}}^{2}+D_{x_{2}}\left(1-\chi(x)\left(1-x_{1}^{2}\right)\right) D_{x_{2}}+D_{x_{3}}^{2},
$$

where $\chi \in C_{0}^{\infty}(B(0,1 / 2)), \chi \leq 1, \chi \equiv 1$ near 0 ; in this case $\varepsilon=\frac{1}{2}$.

Another example is provided by the Laplacian on finite volume noncompact Riemann surfaces, or more generally, certain admissible surfaces considered in [22]. Using the pseudo-Laplacian of Lax and Phillips and Colin de Verdiere [3], our assumptions are satisfied (after a slight modification) with $n=1$ and $\tilde{n}=2$. Since for a generic admissible surface the resonances satisfy the Weyl law with the exponent 2 [22], the order of growth given by Theorem 1.2 is optimal in that case.

\section{OUTLINE OF THE PROOF}

It is well known that the scattering poles, which are also the poles of the meromorphic continuation of the resolvent [13], can be identified with resonances, which in turn are the eigenvalues of nonselfadjoint complex scaled operators. Here, we are concerned with the large angle complex scaling that will allow us to capture all the scattering poles for compactly supported perturbations.

Since outside of a compact region $P$ can be considered a holomorphic partial differential operator $\sum_{j=1}^{n} D_{z_{j}}^{2}$ on $\mathbb{C}^{n}$, we can restrict $P$ to totally real deformations of $\mathbb{R}^{n} \subset \mathbb{C}^{n}$ keeping the compact region fix. We will be interested in deformations $\Gamma_{\theta}$ such that

$$
\Gamma_{\theta} \cap\left\{|z|>R_{1}\right\}=\widehat{\Gamma}_{\theta} \cap\left\{|z|>R_{1}\right\}, \quad \text { where } \widehat{\Gamma}_{\theta}=\left\{e^{i \theta} x: x \in \mathbb{R}^{n}\right\} .
$$

As will be presented in $\S 3$, this produces an operator $P_{\theta}$ on $\Gamma_{\theta}$. We also define

$$
\widetilde{P}_{\theta}=-\Delta_{\theta}, \quad \widehat{P}_{\theta}=-e^{-2 i \theta} \Delta,
$$

which are the restrictions of $\Delta_{z}$ to $\Gamma_{\theta}, \widehat{\Gamma}_{\theta}$ respectively (to make sense of the 'restriction' here we use the almost analytic extension; see $\S 3$ ).

To clarify the relation between $P_{\theta}$ and the scattering poles we shall give an elementary discussion of the one-dimensional case. Let us consider the operator on the line $P=D_{x}^{2}+V(x), V \in L_{\text {comp }}^{1}(\mathbb{R})$, supp $V \subset[a, b]$. The scattering poles $\lambda$ enjoy the following simple characterization (see, e.g., [27]): There exists $u \in C(\mathbb{R})$ such that for some $A \in \mathbb{C}$

$$
\left(D_{x}^{2}+V(x)-\lambda^{2}\right) u=0, \quad u= \begin{cases}e^{i \lambda x}, & x>b, \\ A e^{-i \lambda x}, & x<a .\end{cases}
$$




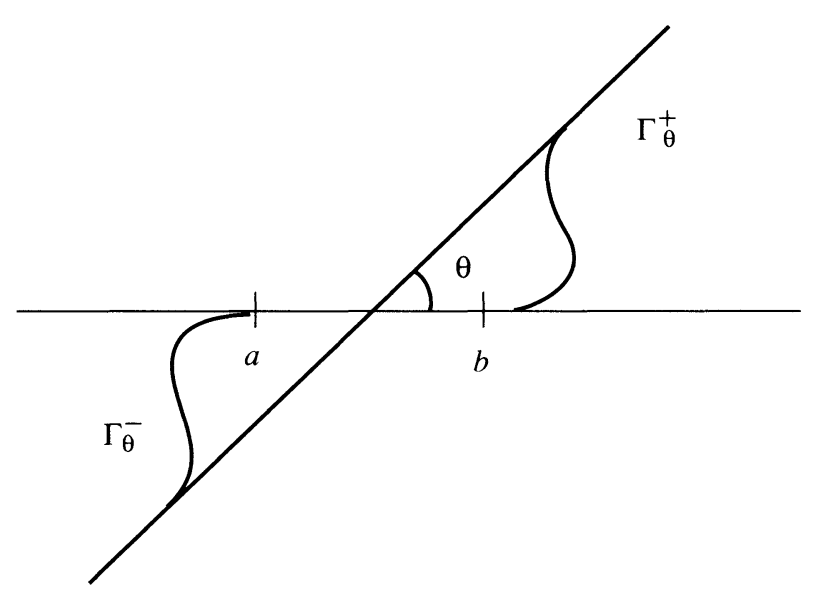

FIGURE 2.1

Note that this characterization does not include the half-bound state $\lambda=0$ and that the multiplicity of the pole is always one.

Let us consider the following smooth deformations of $\mathbb{R}$ (see Figure 2.1)

$$
\Gamma_{\theta}=\Gamma_{\theta}^{-} \cup[a-\varepsilon, b+\varepsilon] \cup \Gamma_{\theta}^{+}
$$

with (2.1) satisfied. Since $V$ is supported in the real part of the contour, we can (using almost analytic extensions) define

$$
P_{\theta}=\left.\left(D_{z}^{2}+V(x)\right)\right|_{\Gamma_{\theta}}, \quad \widetilde{P}_{\theta}=\left.D_{z}^{2}\right|_{\Gamma_{\theta}} .
$$

Now we see that $\left.u\right|_{\Gamma_{0}^{+}}=\left.e^{i \lambda z}\right|_{\Gamma_{0}^{+}},\left.u\right|_{\Gamma_{0}^{-}}=\left.A e^{-i \lambda z}\right|_{\Gamma_{0}^{-}}$and if we define $u_{\theta} \in C\left(\Gamma_{\theta}\right)$ by

$$
u_{\theta}(y)= \begin{cases}u(y), & y \in[a-\varepsilon, b+\varepsilon], \\ e^{i \lambda z}, & y \in \Gamma_{\theta}^{+}, \\ A e^{-i \lambda z}, & y \in \Gamma_{0}^{-},\end{cases}
$$

we obtain a solution

$$
\left(P_{\theta}-\lambda^{2}\right) u_{\theta}=0 .
$$

This is the elementary analogue of Lemma 3.1 with differences coming from the fact that $S^{0}$ is not connected. If we take

$$
\pi-\arg \lambda>\theta>-\arg \lambda,
$$

we easily see that $u_{\theta} \in L^{2}\left(\Gamma_{\theta}\right)$, i.e., $\lambda^{2}$ is an eigenvalue of the (nonselfadjoint) operator $P_{\theta}$.

Assume now that $\lambda^{2}$ is an eigenvalue of $P_{\theta}$. Since for $|z|>R_{1}$ the operator on $\Gamma_{\theta}$ is just

$$
\widehat{P}_{\theta}=\left.D_{z}^{2}\right|_{\widetilde{\Gamma}_{\theta}}, \quad \widehat{\Gamma}_{\theta}=e^{i \theta} \mathbb{R},
$$




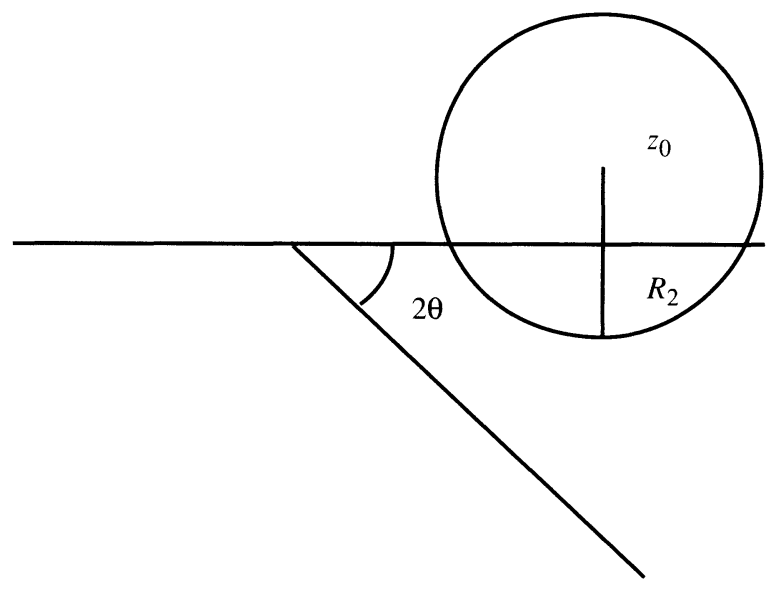

FIGURE 2.2

the uniqueness of solutions for ordinary differential equations shows that the eigenfunction is of the form (2.3). This, however, produces $u$ satisfying (2.2), so that $\lambda$ is a scattering pole.

The eigenvalues of $P_{\theta}, \theta \geq \pi / 4$, will give the scattering poles in $-\pi / 4<$ $\arg \lambda<0$. To estimate the number of scattering poles one uses the classical estimate of Weyl (see \$7) relating the growth of eigenvalues of nonselfadjoint operators to the growth of its characteristic values.

The principal symbol of $P_{\theta}$ takes its values in the angle

$$
-2 \theta-\varepsilon<\arg z<0,
$$

and we define $\sigma_{\text {ess }}\left(P_{\theta}\right)$ to be $e^{-2 i \theta} \overline{\mathbb{R}}_{+}$. Thus for $z_{0} \in \mathbb{C}, \Im z_{0}>0, \Re z_{0}>0$,

$$
P_{\theta}-z_{0} \text { is uniformly elliptic, }
$$

and for large $z_{0}$, it is bounded away from 0 . The characteristic values of $P_{\theta}$ are the eigenvalues of the selfadjoint operator $B_{\theta}^{1 / 2}=\left[\left(P_{\theta}-z_{0}\right)^{*}\left(P_{\theta}-z_{0}\right)\right]^{1 / 2}$, and, roughly speaking, we can estimate the number of eigenvalues of $P_{\theta}-z_{0}$ in a disc of radius $R_{1}$ by the number of characteristic values in an interval $\left[0, R_{2}\right]$ for $R_{2}>R_{1}$. The essential components in that are the Weyl inequality mentioned above, a uniform (in $z_{0}$ ) boundedness of $B_{\theta}^{1 / 2}$ away from 0 and the Weyl-type estimate on the number of eigenvalues of $B_{\theta}^{1 / 2}$. The last estimate is possible only if the disc $D\left(0, R_{2}\right)$ does not intersect the essential spectrum of $P_{\theta}-z_{0}, \sigma_{\text {ess }}\left(P_{\theta}-z_{0}\right)=e^{-2 i \theta} \mathbb{R}_{+}-z_{0}$; see Figure 2.2. For $\theta>\pi / 4$ the discs $D\left(z_{0}, R\right)$ that have to avoid $e^{-2 i \theta} \overline{\mathbb{R}}_{+}$can only cover the fourth open quadrant.

To allow for large angles we need operators that will have the discrete spectrum related to that of $P_{\theta}$ but contained in a smaller angle around the real axis. This is achieved by taking a cubic root of $P_{\theta},\left(P_{\theta}-\omega_{0}\right)^{1 / 3}$. Here, we carry out the same programme as outlined above for these operators. Section 3 
gives the theory of large angle complex scaling. Sections 4 and 5 are devoted to the analysis of the cubic root for our class of operators. In particular, we are interested in the localization of our functional calculus. That is used in $\S 6$ to study compactly supported functions of the analogue of the operator $B_{\theta}$ above. Finally, in $\S 7$, the procedure outlined above for $P_{\theta}$ yields Theorem 1.2.

To obtain Corollary 1.1 we need to check (1.7) with $\tilde{n}=n$. This follows, however, from the following estimate on the spectral function of $P$ :

$$
|e(\lambda, x, x)| \leq C_{R} \lambda^{n / 2}, \quad|x| \leq R,
$$

which is, with a slight modification to our case, the contents of [9, Theorem 17.5.3].

\section{REVIEW OF COMPLEX SCALING}

We start by discussing the restriction of differential operators with holomorphic coefficients to a totally real submanifold of maximal dimension. A smooth submanifold $\Gamma \subset \mathbb{C}^{n}$ is called totally real if

$$
T_{x} \Gamma \cap i T_{x} \Gamma=\{0\} \quad \text { for every } x \in \Gamma \text {. }
$$

Here $i$ denotes the natural action on tangent vectors induced by the multiplication by the imaginary unit. Clearly $\operatorname{dim}_{\mathbb{R}} \Gamma \leq n$ if $\Gamma$ is totally real, and the natural example of a totally real manifold of maximal dimension $n$ is $\Gamma=\mathbb{R}^{n}$.

If $\Gamma$ is totally real and if $f$ is a smooth map from a complex neighbourhood of a point $x_{0} \in \Gamma$ into $\mathbb{C}^{n}$ and $d f\left(x_{0}\right)$ is injective and $\bar{\partial}_{x} f\left(x_{0}\right)=0$, then $d f\left(x_{0}\right)$ is complex linear and invertible, and hence $f(\Gamma)$ is a totally real submanifold of $\mathbb{C}^{n}$ near $f\left(x_{0}\right)$, at least after restricting $\Gamma$ to some neighbourhood of $x_{0}$.

Let $\Gamma \subset \mathbb{C}^{n}$ be smooth and of real dimension $n$. Then locally we can represent $\Gamma$ as $f\left(\mathbb{R}^{n}\right)$, where $f: \mathbb{R}^{n} \rightarrow \mathbb{C}^{n}$ is smooth with injective differential. Let $\tilde{f}$ be an almost analytic extension of $f$ so that $\bar{\partial} \tilde{f}$ vanishes to infinite order on $\mathbb{R}^{n}$. Let $y \in \mathbb{R}^{n}, f(y) \in \Gamma$. Then since $d \tilde{f}(y)$ is complex linear, $i T_{f(y)} \Gamma=d \tilde{f}(y)\left(i T_{y} \mathbb{R}^{n}\right)$. Hence $\Gamma$ is totally real in a neighbourhood of $f(y)$ if and only if $d \tilde{f}(y)\left(T_{y} \mathbb{R}^{n}\right) \cap d \tilde{f}(y)\left(i T_{y} \mathbb{R}^{n}\right)=\{0\} ;$ that is, if and only if $d \tilde{f}(x)$ is injective, or more explicitly, if and only if

$$
\operatorname{det}\left(\partial_{y_{k}} f_{j}(y)\right) \neq 0 \text {. }
$$

Let $\Omega \subset \mathbb{C}^{n}$ be open, and let $\Gamma \subset \Omega$ be a totally real submanifold of maximal dimension. Let $P\left(x, D_{x}\right)$ be a partial differential operator of order $m$ with holomorphic coefficients on $\Omega$,

$$
P\left(x, D_{x}\right)=\sum_{|\alpha| \leq m} a_{\alpha}(x) D_{x}^{\alpha}
$$

where $D_{x}=i^{-1} \partial_{x}, \partial_{x}=\left(\partial_{x_{1}}, \ldots, \partial_{x_{n}}\right), \partial_{x_{j}}=\frac{1}{2}\left(\partial_{\Re x_{j}}+i^{-1} \partial_{\Im x_{j}}\right)$. We then define $P_{\Gamma}: C^{\infty}(\Gamma) \rightarrow C^{\infty}(\Gamma)$ by

$$
P_{\Gamma} u=\left.\left(P\left(x, D_{x}\right) \tilde{u}\right)\right|_{\Gamma}
$$


where $\tilde{u}$ is an almost analytic extension of $u$, that is, a smooth extension of $u$ to a neighbourhood of $\Gamma$ such that $\bar{\partial} \tilde{u}$ vanishes to infinite order on $\Gamma$. (With $f$ and $\tilde{f}$ as above, we can take $\tilde{u}=(\widetilde{u \circ f}) \circ \tilde{f}^{-1}$, where $\widetilde{u \circ f}$ is an almost analytic extension of $u \circ f$. Notice that $\bar{\partial}\left(\tilde{f}^{-1}\right)$ vanishes to infinite order on $\Gamma$.) Since $\tilde{u}$ is unique up to a smooth function that vanishes to infinite order on $\Gamma$, the definition of $P_{\Gamma}$ is unambiguous. Let us check that $P_{\Gamma}$ is a differential operator on $\Gamma$ and compute it using the local parametrization $f$. We write

$$
\begin{aligned}
& \partial_{y_{j}}=\sum_{k}\left(\partial_{y_{j}} \tilde{f}_{k}\right) \partial_{x_{k}}+\sum_{k}\left(\partial_{y_{j}} \overline{\tilde{f}}_{k}\right) \partial_{\bar{x}_{k}}, \\
& \partial_{\bar{y}_{j}}=\sum_{k}\left(\partial_{\bar{y}_{j}} \tilde{f}_{k}\right) \partial_{x_{k}}+\sum_{k}\left(\partial_{\bar{y}_{j}} \overline{\tilde{f}}_{k}\right) \partial_{\bar{x}_{k}},
\end{aligned}
$$

recall that $\bar{\partial}_{y} \tilde{f}=\mathscr{O}\left(|\Im y|^{\infty}\right)$, and conclude that

$$
\left(P\left(x, D_{x}\right) \tilde{u}\right) \circ \tilde{f}(y)=\sum_{|\alpha| \leq m} a_{\alpha}(f(y))\left({ }^{t}\left(\partial_{y} \tilde{f}\right)^{-1} D_{y}\right)^{\alpha}(\tilde{u} \circ f)+\mathscr{O}\left(|\Im y|^{\infty}\right) ;
$$

if we also use the almost analyticity of $\tilde{u} \circ f$, we get

$$
\begin{gathered}
\left(P_{\Gamma} u\right) \circ f=Q\left(y, D_{y}\right)(u \circ f), \\
Q\left(y, D_{y}\right)=\sum_{|\alpha| \leq m} a_{\alpha} \circ f\left({ }^{t}\left(\partial_{y} \tilde{f}\right)^{-1} D_{y}\right)^{\alpha},
\end{gathered}
$$

where $D_{y}$ now means $i^{-1}$ times $y$-derivation in the standard real sense. The principal symbol $q$ of $Q$ is given by the usual relation,

$$
q(y, \eta)=p\left(f(y),{ }^{t} f^{\prime}(y)^{-1} \eta\right),
$$

where $p$ is the principal symbol of $P$.

More invariantly, if we identify $T^{*} \Gamma$ with a submanifold of $\mathbb{C}^{n} \times \mathbb{C}^{n}$ via the map $T^{*} \Gamma \ni(x, d u(x)) \mapsto\left(x, \partial_{x} \tilde{u}(x)\right) \in \mathbb{C}^{n} \times \mathbb{C}^{n}$ for $u$ smooth and real, then the principal symbol of $P_{\Gamma}$ is given by $p_{\Gamma}=\left.p\right|_{T^{*} \Gamma}$. We need a deformation result:

Lemma 3.1. Let $\omega \subset \mathbb{R}^{n}$ be open, and let $f:[0,1] \times \omega \ni(t, y) \mapsto f(t, y) \in \mathbb{C}^{n}$ be a smooth proper map such that $\operatorname{det}\left(\partial_{y} f(t, y)\right) \neq 0$ for all $(t, y)$ and such that $f(t, \cdot)$ is injective. Assume that $f(t, y)=f(0, y)$ for $y \in \omega \backslash K$ where $K$ is some compact subset of $\omega$. Let $P\left(x, D_{x}\right)$ be a partial differential operator with holomorphic coefficients defined in a neighbourhood of $f([0,1] \times \omega)$ such that $P_{\Gamma_{t}}$ is elliptic for $0 \leq t \leq 1$. Here $\Gamma_{t}=f(\{t\} \times \omega)$. If $u_{0} \in \mathscr{D}^{\prime}\left(\Gamma_{0}\right)$ and $P_{\Gamma_{0}} u_{0}$ extends to a holomorphic function in a neighbourhood of $f([0,1] \times \omega)$, then the same thing holds for $u_{0}$.

Proof. We shall use a version of the FBI-transform. Let $\Gamma$ be totally real of maximal dimension, and let $x_{0} \in \Gamma$. Then, as we shall show later, if $u \in \mathscr{D}^{\prime}(\Gamma)$, we have for $x$ in a neighbourhood of $x_{0}, \Sigma_{0}$ say, which does not depend 
on $u$,

$$
u(x)=\int_{\Gamma \times\left(\left.T^{*} \Gamma\right|_{\Sigma_{0}}\right)} e^{i \varphi(x, y, \alpha)} a(x, y, \alpha) \chi(y) u(y) d y d \alpha+K u(x),
$$

where $d y=\left.d y_{1} \wedge \cdots \wedge d y_{n}\right|_{\Gamma}, d \alpha$ is the canonical volume element on $T^{*} \Gamma$, $K u$ has a holomorphic extension to a $u$-independent neighbourhood of $x_{0}$. Moreover $\varphi(x, y, \alpha)$ is positively homogeneous of degree 1 in $\alpha_{\xi}$ with the natural notation $\alpha=\left(\alpha_{x}, \alpha_{\xi}\right)$ and has a holomorphic extension to a neighbourhood of $\left\{\left(x_{0}, x_{0}\right)\right\} \times\left(T_{x_{0}}^{*} \Gamma \backslash\{0\}\right)$ in $\mathbb{C}^{n} \times \mathbb{C}^{n} \times \mathbb{C}^{n} \times\left(\mathbb{C}^{n} \backslash 0\right)$ (when viewing $T^{*} \Gamma$ as a subset of $\mathbb{C}^{n} \times \mathbb{C}^{n}$ as above). We also have $\varphi(x, y, \alpha)=(x-y) \alpha_{\xi}+$ $\mathscr{O}\left(\left(x-\alpha_{x}\right)^{2}+\left(y-\alpha_{x}\right)^{2}\right)$ for $\left|\alpha_{\xi}\right|=1$ and $\Im \varphi(x, y, \alpha) \sim\left|x-\alpha_{x}\right|^{2}+\left|y-\alpha_{x}\right|^{2}$ for $x, y \in \Gamma, \alpha \in T^{*} \Gamma,\left|\alpha_{\xi}\right|=1 . a(x, y, \alpha)$ is an analytic symbol of order $n / 2$ (with $\alpha_{\xi}$ as the fiber variables), and $\chi$ is a cutoff function equal to 1 in a neighbourhood of $x_{0}$.

Using the ellipticity of $P$, we can construct an analytic symbol $b(x, y, \alpha)$ of order $n / 2-m$ such that

$$
{ }^{t} P\left(y, D_{y}\right)\left(e^{i \varphi(x, y, \alpha)} b(x, y, \alpha)\right)=e^{i \varphi(x, y, \alpha)}(a(x, y, \alpha)+r(x, y, \alpha)),
$$

where $r$ is holomorphic for $x, y$ in a neighbourhood of $x_{0}$ and of exponential decrease,

$$
|r(x, y, \alpha)| \leq C e^{-\left|\alpha_{\xi}\right| / C}
$$

for some $C>0$.

Using this in (3.8), we get after an integration by parts (justified by the Stokes formula),

$$
u(x)=\int e^{i \varphi(x, y, \alpha)} b(x, y, \alpha) \chi(y) P u(y) d y d \alpha+\widetilde{K} u(x),
$$

where $\widetilde{K}$ has the same properties as $K$ in (3.8). If we now assume that $P_{\Gamma} u$ has a holomorphic extension to some arbitrary but fixed neighbourhood $\Omega$ of $x_{0}$, then by using the Stokes formula and deformation of integration contours, we see that the integral in (3.11) extends holomorphically to some neighbourhood of $x_{0}$ that depends on $\Omega$ but not on $u$.

Using this fact and a simple compactness argument, we get the lemma.

It remains to establish the representation (3.8), which, as well as the lemma itself, is essentially well known (see for instance [6, Proposition 4.1] or [15]). We start with the case $\Gamma=\mathbb{R}^{n}$. Then (see [14] or $[9, \S 9.6]$ ) we can represent the $\delta$-function at the origin as

$$
\delta(x)=\int e^{i\left(\langle x, \xi\rangle+i \lambda|\xi| x^{2} / 2\right)} b_{\lambda}(x, \xi) d \xi
$$

for any $\lambda>0$, where $b_{\lambda}=(2 \pi)^{-n}(1+i \lambda\langle x, \xi\rangle /(2|\xi|))$ is a classical analytic symbol. (To see this simply make the change of variables $\eta=\xi+i \lambda|\xi| x / 2$ 
in $\delta(x)=(2 \pi)^{-n} \int e^{i\langle\eta, x\rangle} d \eta$. ) Replacing $x$ by $x-y$ in (3.12) we obtain a representation of the identity operator, and by adding $n$ more variables we arrive at

$$
u(x)=\int_{\alpha \in \mathbb{R}^{2 n}} \int_{y \in \mathbb{R}^{n}} e^{i \phi_{\lambda}(x, y, \alpha)} a_{\lambda}(x, y, \alpha) u(y) d y d \alpha,
$$

with $\phi_{\alpha}(x, y, \alpha)=\left\langle x-y, \alpha_{\xi}\right\rangle+i \lambda\left[\left(x-\alpha_{x}\right)^{2}+\left(y-\alpha_{x}\right)^{2}\right]$ and $a_{\lambda}(x, y, \alpha)=$ $c_{n} \lambda^{-n / 2}\left(x-y, \alpha_{\xi}\right)$ (the variable of integration $\alpha_{x}$ is removed by computing the Gaussian integral). Restricting the $\alpha_{x}$-domain of integration will produce an error $K u$ in (3.8), and thus it is established in the case $\Gamma=\mathbb{R}^{n}$.

We now pass to the case of general $\Gamma$, and we may assume that $x_{0}=0$ and $T_{0} \Gamma=\mathbb{R}^{n}$. Choosing $\lambda>0$ sufficiently large, we may try to take $\varphi=\varphi_{\lambda}$ and $a=a_{\lambda}$ in (3.8). Let us denote the first term on the right-hand side of (3.8) with these $\varphi$ and $a$ by $A u$. Using the Stokes formula we can change the integration contour in $A u$ into

$$
y \in \Gamma, \quad \alpha_{x} \in \Gamma \cap \Sigma_{0}, \quad \alpha_{\xi} \in T_{y}^{*}(\Gamma),
$$

introducing errors of the type " $K u$ " only. After that, again modulo terms of the type " $K u$," we can eliminate integration in $\alpha_{x}$ as in the case of $\mathbb{R}^{n}$ and get

$$
A u(x) \equiv \int_{y \in \Gamma} \int_{\xi \in T_{y}^{*} \Gamma} e^{i\left(\langle x-y, \xi\rangle+\frac{i}{2} \lambda\left(\xi^{2}\right)^{1 / 2}(x-y)^{2}\right)} b_{\lambda}(x-y, \xi) d \xi u(y) d y .
$$

Let us denote the right-hand side above by $\widetilde{A} u$. By analytic continuation from (3.12) we see that the distribution kernel of $\tilde{A}$ vanishes outside the diagonal. Since it is a pseudodifferential operator of order 0 , it has to be of the form $\widetilde{A} u(x)=\tilde{a}(x) u(x)$. Testing against oscillatory functions and using deformations of contours together with (3.12), we see that $\tilde{a}(x) \equiv 1$, and the proof of (3.8) is complete.

Let us recall our complex Hilbert space $\mathscr{H}$ with an orthogonal decomposition,

$$
\mathscr{H}=\mathscr{H}_{R_{0}} \oplus L^{2}\left(\mathbb{R}^{n} \backslash B\left(0, R_{0}\right)\right) .
$$

Here $R_{0}>0$ is some fixed constant and $B(x, R)$ denotes the open ball in $\mathbb{R}^{n}$ with center $x$ and radius $R$. If $f \in L^{\infty}\left(\mathbb{R}^{n}\right)$ is constant on $B\left(0, R_{0}\right)$ and if $u \in \mathscr{H}$, then there is a natural way to define $f u \in \mathscr{H}$, and the "multiplication" operator $\mathscr{H} \ni u \mapsto f u \in \mathscr{H}$ is bounded of norm $\leq\|f\|_{L^{\infty}}$ (actually with equality if $\mathscr{H}_{R_{0}}$ is nonempty). We have $f(g u)=(f g) u,(f+g) u=f u+g u$. If $f=\mathbb{1}_{F}$ is the characteristic function of some measurable set $F$ that either contains $B\left(0, R_{0}\right)$ or is disjoint from $B\left(0, R_{0}\right)$, then for $u \in \mathscr{H}$ we define $\left.u\right|_{F}=\mathbb{1}_{F} u$.

If $\chi \in C^{2}\left(\mathbb{R}^{2}\right)$ is constant $=\chi_{0}$ near $\overline{B\left(0, R_{0}\right)}$ and with bounded derivatives up to the order 2 , then if $u \in \mathscr{D}$, we have $\chi u \in \mathscr{D}$ and $P \chi u=\chi P u-$ $[\Delta, \chi] u$, where the last term is defined in the obvious way with a vanishing $\mathscr{H}_{R_{0}}$-component. To see this we write $\chi u=\chi_{0} u+\left(\chi-\chi_{0}\right) u$ and use (1.4), (1.5). 
As we shall see below, it follows that $P$ has only discrete spectrum on ]$-\infty, 0[$.

For a given $\varepsilon_{0}$ and $a_{0}, 0<\varepsilon_{0}<\pi / 2, a_{0}>0$, we can construct a smooth function $[0, \pi] \times\left[0, \infty\left[\ni(\theta, t) \mapsto f_{\theta}(t) \in \mathbb{C}\right.\right.$, injective for every $\theta$, with the following properties:

(i) $f_{\theta}(t)=t$ for $0 \leq t \leq R_{0}+a_{0}$;

(ii) $0 \leq \arg f_{\theta}(t) \leq \theta, 0 \leq \arg \partial_{t} f_{\theta}(t) \leq \theta+\varepsilon_{0}, \partial_{t} f_{\theta} \neq 0$;

(iii) $\arg f_{\theta}(t) \leq \arg \partial_{t} f_{\theta}(t) \leq \arg f_{\theta}(t)+\varepsilon_{0}$;

(iv) $f_{\theta}(t)=e^{i \theta} t$ for $t \geq T_{0}$, where $T_{0}$ only depends on $\varepsilon_{0}$ and $a_{0}$.

Let us identify $\mathbb{R}^{n} \backslash(0)$ with $] 0, \infty\left[\times \mathbb{S}^{n-1}\right.$ by means of standard "polar coordinates": $x \mapsto(t, \omega), t=|x|, \omega=x /|x|, x=t \omega$, and consider the maps

$$
\kappa_{\theta}:(t, \omega) \mapsto f_{\theta}(t) \omega .
$$

We also consider $\kappa_{\theta}$ as a map: $\mathbb{R}^{n} \rightarrow \mathbb{C}^{n}$ in the obvious way. Let $\Gamma_{\theta}=\kappa_{\theta}\left(\mathbb{R}^{n}\right)$. Identifying $\mathbb{C}^{n}$ locally near a point of $\Gamma_{\theta} \backslash\{0\}$ with $\left\{(s, w) \in \mathbb{C} \times \mathbb{C}^{n} ; \sum w_{j}^{2}=\right.$ 1) (using the substitution $x=s w$ ), we see that $\Gamma_{\theta}$ is a totally real submanifold of maximal dimension. We can define naturally a dilated operator $P_{\theta}$ in the following way: We work in the Hilbert space

$$
\mathscr{H}_{\theta}=\mathscr{H}_{R_{0}} \oplus L^{2}\left(\Gamma_{\theta} \backslash B\left(0, R_{0}\right)\right),
$$

where $B\left(0, R_{0}\right)$ denotes the real ball as before. Actually, we shall mostly consider $\mathscr{H}_{\theta}$ as a Banach space (and the dual will be identified with $\mathscr{H}_{-\theta}$ ). If $\chi \in C_{0}^{\infty}\left(B\left(0, R_{0}+a_{0}\right)\right)$ is equal to 1 in a neighbourhood of $\overline{B\left(0, R_{0}\right)}$, we put

$$
\mathscr{D}_{\theta}=\left\{u \in \mathscr{H}_{\theta} ; \chi u \in \mathscr{D},(1-\chi) u \in H^{2}\left(\Gamma_{\theta} \backslash B\left(0, R_{0}\right)\right)\right\} \text {. }
$$

This definition does not depend on the choice of $\chi$. We let $P_{\theta}$ be the unbounded operator $\mathscr{H}_{\theta} \rightarrow \mathscr{H}_{\theta}$ with domain $\mathscr{D}_{\theta}$ defined by

$$
\begin{aligned}
\left.P_{\theta} u\right|_{B\left(0, R_{0}\right)} & =\left.P(\chi u)\right|_{B\left(0, R_{0}\right)}, \\
\left.P_{\theta} u\right|_{\Gamma_{\theta} \backslash B\left(0, R_{0}\right)} & =-\Delta_{\Gamma_{\theta}}\left(\left.u\right|_{\Gamma_{\theta} \backslash B\left(0, R_{0}\right)}\right), \quad u \in \mathscr{D}_{\theta} .
\end{aligned}
$$

Again this definition does not depend on the choice of $\chi$ above.

In the $(s, w)$-coordinates we have $-\Delta=D_{s}^{2}+s^{-1}(n-1) i D_{s}+s^{-2} D_{w}^{2}$, where $-D_{w}^{2}$ simply denotes the natural Laplacian in the $w$-variables. Outside $B\left(0, R_{0}\right)$ we can express $P_{\theta}$ in polar coordinates,

$$
P_{\theta}=\left(f^{\prime}(t)^{-1} D_{t}\right)^{2}+\left(f(t) f^{\prime}(t)\right)^{-1}(n-1) i D_{t}+(f(t))^{-2} D_{\omega}^{2},
$$

where $-D_{\omega}^{2}$ is the Laplacian on $\mathbb{S}^{n-1}$. The principal symbol of $P_{\theta}$ is then for $t>R_{0}$

$$
p_{\theta}=\left(\tau / f^{\prime}(t)\right)^{2}+\left(\omega^{*} / f(t)\right)^{2},
$$

where $\omega^{* 2}$ denotes the principal symbol of $D_{\omega}^{2}$. We see that for each fixed $t$, $p_{\theta}$ is elliptic and takes its values in an angle of size less than $\pi-2 \varepsilon_{0}$. When we 


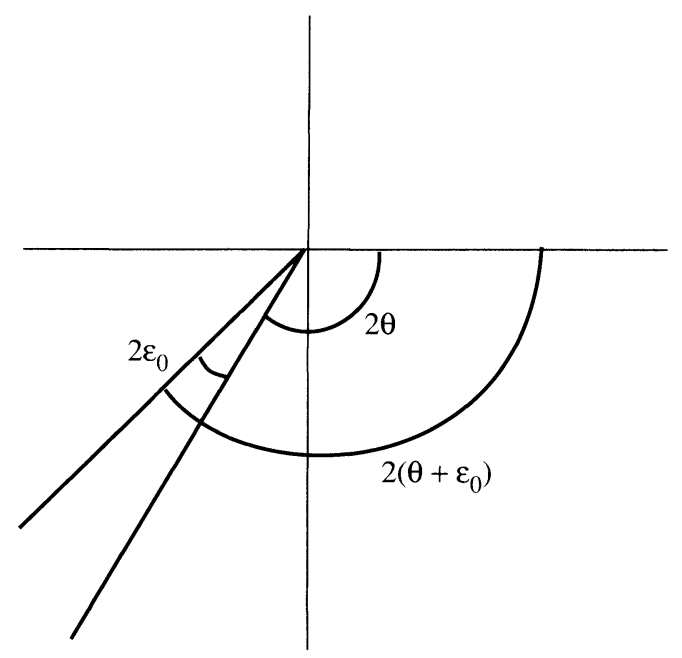

FIGURE 3.1

vary $t$ we see that $p_{\theta}$ takes its value in the angle of size $2\left(\theta+\varepsilon_{0}\right)$; see Figure 3.1 .

Notice also that ii we identify $\Gamma_{\theta}$ with $\mathbb{R}^{n}$ by means of $\kappa_{\theta}$ then $P_{\theta}$ is equal to $-e^{-2 i \theta} \Delta$ outside a ball (in $\mathbb{R}^{n}$ ) of radius $T_{0}$.

Lemma 3.2. If $z \in \mathbb{C} \backslash\{0\}, \arg z \neq-2 \theta$, then $P_{\theta}-z: \mathscr{D}_{\theta} \rightarrow \mathscr{H}_{\theta}$ is a Fredholm operator.

Proof. We shall invert $P_{\theta}-z$ modulo compact operators. On the submanifold $\Gamma_{\theta}$ we introduce a smooth partition of unity: $1=\theta_{1}+\theta_{2}+\theta_{3}$ with $\operatorname{supp} \theta_{1} \subset$ $B\left(0, R_{0}+a_{0}\right), \operatorname{supp} \theta_{3}$ contained in the region where $P_{\theta}=-e^{-2 i \theta} \Delta=_{\text {def }}-\Delta_{\theta}$, $\operatorname{supp} \theta_{2}$ compact and disjoint from $\overline{B\left(0, R_{0}\right)}$. Let $\chi_{j}$ have the same properties as the $\theta_{j}$ except that they do not form a partition of unity, and with the property that $\chi_{j}=1$ in a neighbourhood of the support of $\theta_{j}$. Let $z_{0} \in \mathbb{C} \backslash \mathbb{R}$, and put

$$
E(z)=\chi_{1}\left(P-z_{0}\right)^{-1} \theta_{1}+Q \theta_{2}+\chi_{3}\left(-\Delta_{\theta}-z\right)^{-1} \theta_{3},
$$

where $Q$ is a properly supported parametrix of the elliptic operator $P_{\theta}-z$ in the complement of $B\left(0, R_{0}\right)$. Then we get

$$
\left(P_{\theta}-z\right) E(z)=I+K(z),
$$

where

$$
\begin{aligned}
K(z)= & \left(z_{0}-z\right) \chi_{1}\left(P-z_{0}\right)^{-1} \theta_{1}+\left[P_{\theta}, \chi_{1}\right]\left(P-z_{0}\right)^{-1} \theta_{1} \\
& +\left(\left(P_{\theta}-z\right) Q-I\right) \theta_{2}+\left[P_{\theta}, \chi_{3}\right]\left(-\Delta_{\theta}-z\right)^{-1} \theta_{3} .
\end{aligned}
$$

Using the exponential decay of the kernel of $\left(-\Delta_{\theta}-z\right)^{-1}$ away from the diagonal and assumptions $(1.4),(1.6)$, we see that $K(z)$ is compact: $\mathscr{H}_{\theta} \rightarrow \mathscr{H}_{\theta}$. 
As an approximate left inverse, we put

$$
F(z)=\theta_{1}\left(P-z_{0}\right)^{-1} \chi_{1}+\theta_{2} Q+\theta_{3}\left(-\Delta_{\theta}-z\right)^{-1} \chi_{3} .
$$

Then

$$
F\left(P_{\theta}-z\right)=1+L(z)
$$

where

$$
\begin{aligned}
L(z)= & \left(z_{0}-z\right) \theta_{1}\left(P-z_{0}\right)^{-1} \chi_{1}-\theta_{1}\left(P-z_{0}\right)^{-1}\left[P_{\theta}, \chi_{1}\right] \\
& +\theta_{2}\left(Q\left(P_{\theta}-z\right)-I\right)-\theta_{3}\left(-\Delta_{\theta}-z\right)^{-1}\left[P_{\theta}, \chi_{3}\right] .
\end{aligned}
$$

We shall verify that $L(z)$ is compact both as an operator $\mathscr{H}_{\theta} \rightarrow \mathscr{H}_{\theta}$ and as an operator $\mathscr{D}_{\theta} \rightarrow \mathscr{D}_{\theta}$. We denote the terms of the right-hand side of the last equation by $I, I I, I I I$, and $I V$. The term $I$ is clearly compact in $\mathscr{H}_{\theta}$. That $I I$ is compact in $\mathscr{H}_{\theta}$ follows if we write $\left(P-z_{0}\right)^{-1}\left[P_{\theta}, \chi_{1}\right]=Q_{0}\left[P_{\theta}, \chi_{1}\right]-$ $\left(P-z_{0}\right)^{-1}\left(\left(P-z_{0}\right) Q_{0}-1\right)\left[P_{\theta}, \chi_{1}\right]$, where $Q_{0}$ is a properly supported parametrix of $P-z_{0}$ outside $\overline{B\left(0, R_{0}\right)}$. The compactness of $I I I$ and $I V$ in $\mathscr{H}_{\theta}$ and in $\mathscr{D}_{\theta}$ is evident. It remains to establish the compactness of $I$ and $I I$ as operators $\mathscr{D}_{\theta} \rightarrow \mathscr{D}_{\theta}$. We only have to show that $\left(P-z_{0}\right) I$ and $\left(P-z_{0}\right) I I: \mathscr{D}_{\theta} \rightarrow$ $\mathscr{H}_{\theta}$ are compact. We have $\left(P-z_{0}\right) I=\left(z_{0}-z\right)\left(\theta_{1}+\left[P, \theta_{1}\right]\left(P-z_{0}\right)^{-1}\right) \chi_{1}$ and the compactness then follows from (1.4), (1.6). Similarly: $\left(P-z_{0}\right) I I=$ $-\left(\theta_{1}+\left[P_{\theta}, \theta_{1}\right]\left(P-z_{0}\right)^{-1}\right)\left[P_{\theta}, \chi_{1}\right]$ is compact $\mathscr{D}_{\theta} \rightarrow \mathscr{H}_{\theta}$.

The index of $P_{\theta}-z$ is locally constant on $\{(\theta, z) ; 0 \leq \theta<\pi, z \in$ $\mathbb{C} \backslash e^{-2 i \theta}\left[0,+\infty[\}\right.$ and if we first deform $z$ into $e^{i \delta}$ for some very small $\delta>0$ and then $\theta$ down to 0 , we see that the index of $P_{\theta}-z$ is equal to the index of $P-e^{i \delta}$, which is zero since $P$ is selfadjoint. Thus the index of $P_{\theta}-z$ is 0 for $z \in \mathbb{C} \backslash e^{-2 i \theta}[0,+\infty[$. In particular, we have

Lemma 3.3. A point $z \in \mathbb{C} \backslash e^{-2 i \theta}\left[0,+\infty\left[\right.\right.$ belongs to the spectrum of $P_{\theta}$ if and only if $\operatorname{ker}\left(P_{\theta}-z\right) \neq 0$.

We shall next show

Lemma 3.4. Assume that $0 \leq \theta_{1}<\theta_{2}<\pi$, and let $z_{0} \in \mathbb{C} \backslash\left\{r e^{-2 i \theta} ; \quad r \geq 0\right.$, $\left.\theta_{1} \leq \theta \leq \theta_{2}\right\}$. Then $\operatorname{dim} \operatorname{ker}\left(P_{\theta_{1}}-z_{0}\right)=\operatorname{dim} \operatorname{ker}\left(P_{\theta_{2}}-z_{0}\right)$.

Proof. We shall apply Lemma 3.1 to the family $\Gamma_{\theta}$, or rather to a modified family that will satisfy all the assumptions of the lemma. Let $0 \leq \chi \in$ $C_{0}^{\infty}(] \frac{1}{2}, 1 \frac{1}{2}[;[0,1])$ be equal to 1 near 1 , and put for $T \geq 1$

$$
F_{\theta_{1}, \theta_{2}, T}(t)=f_{\theta_{1}}(t)+\chi(t / T)\left(f_{\theta_{2}}(t)-f_{\theta_{1}}(t)\right) .
$$

We let $\Gamma_{\theta_{1}, \theta_{2}, T}$ be the corresponding submanifold of $\mathbb{C}^{n}$, defined as earlier but with $f_{\theta}$ replaced by $F_{\theta_{1}, \theta_{2}, T}$. If $\theta_{2}-\theta_{1}$ is small enough, then Lemma 3.1 is applicable to the family of totally real submanifolds: $[0,1] \ni t \mapsto \Gamma_{\theta_{1}, \theta_{1}+t\left(\theta_{2}-\theta_{1}\right), T}$ and the operator $\Delta$. (Naturally we restrict the attention to the parts of these 
submanifolds that are outside $B\left(0, R_{0}\right)$.) We conclude that if $\left(P_{\theta_{1}}-z_{0}\right) u_{\theta_{1}}=$ $0, u \in \mathscr{D}_{\theta_{1}}$, then $u_{\theta_{1}}$ extends to a holomorphic function in a neighbourhood of $\bigcup_{\theta_{1} \leq \theta \leq \theta_{2}}\left(\Gamma_{\theta} \backslash B\left(0, R_{0}\right)\right)$ and by restriction we get an element $u_{\theta}$ with $\chi u_{\theta} \in \mathscr{D}$, $(1-\chi) u_{\theta} \in C^{\infty}$, which satisfies $\left(P_{\theta}-z_{0}\right) u_{\theta}=0$ in the natural sense. Here $\chi \in C_{0}^{\infty}\left(\mathbb{R}^{n}\right)$ is equal to 1 near $B\left(0, R_{0}\right)$. So far $\theta_{2}-\theta_{1}$ is supposed to be small, but it is enough that $0 \leq \theta_{2}-\theta_{1} \leq \delta_{0}$ for some $\delta_{0}>0$ that is independent of $\theta_{1}$, and hence by iteration we can eliminate the smallness assumption.

So far our argument does not require any assumption on $z_{0}$ since it is based only on the local ellipticity of $P_{\theta}$. We shall now make use of the assumption on $z_{0}$, and we begin with a preliminary observation based on ellipticity at infinity. Let $0<a_{1}<a_{2}<b_{2}<b_{1}$, and put $\Omega_{j}=\left\{y \in \mathbb{R}^{n} ; a_{j}<|y|<b_{j}\right\}, j=1,2$. The change of variables $y=T z$ transforms $-\Delta_{y}$ into $-T^{-2} \Delta_{z}$. For $T \geq 1$, we may view $-T^{-2} e^{-2 i \theta} \Delta_{z}-z_{0}$ as an elliptic semiclassical operator, and hence we have the standard a priori estimate,

$$
\sum_{|\alpha| \leq 2}\left\|\left(T^{-1} D_{z}\right)^{\alpha} u\right\|_{L^{2}\left(\Omega_{2}\right)} \leq C\left(\left\|\left(-T^{-2} e^{-2 i \theta} \Delta_{z}-z_{0}\right) u\right\|_{L^{2}\left(\Omega_{1}\right)}+\|u\|_{L^{2}\left(\Omega_{1} \backslash \Omega_{2}\right)}\right),
$$

with $C$ independent of $T$, and in the $y$-variables we get

$$
\begin{aligned}
& \sum_{|\alpha| \leq 2}\left\|\left(D_{y}\right)^{\alpha} u\right\|_{L^{2}\left(T \Omega_{2}\right)} \\
& \quad \leq C\left(\left\|\left(-e^{-2 i \theta} \Delta_{y}-z_{0}\right) u\right\|_{L^{2}\left(T \Omega_{1}\right)}+\|u\|_{L^{2}\left(T\left(\Omega_{1} \backslash \Omega_{2}\right)\right)}\right) .
\end{aligned}
$$

Outside a bounded set the restriction of $-\Delta$ to $\Gamma_{\theta_{1}, \theta_{2}, T}$ is a small perturbation of $-e^{-2 i \theta} \Delta_{y}$ when $\theta_{2}-\theta_{1}$ is small and (3.19) still applies to that operator. We choose $T \Omega_{1}$ and $T \Omega_{2}$ according to Figure 3.2 where only the $s$-variables

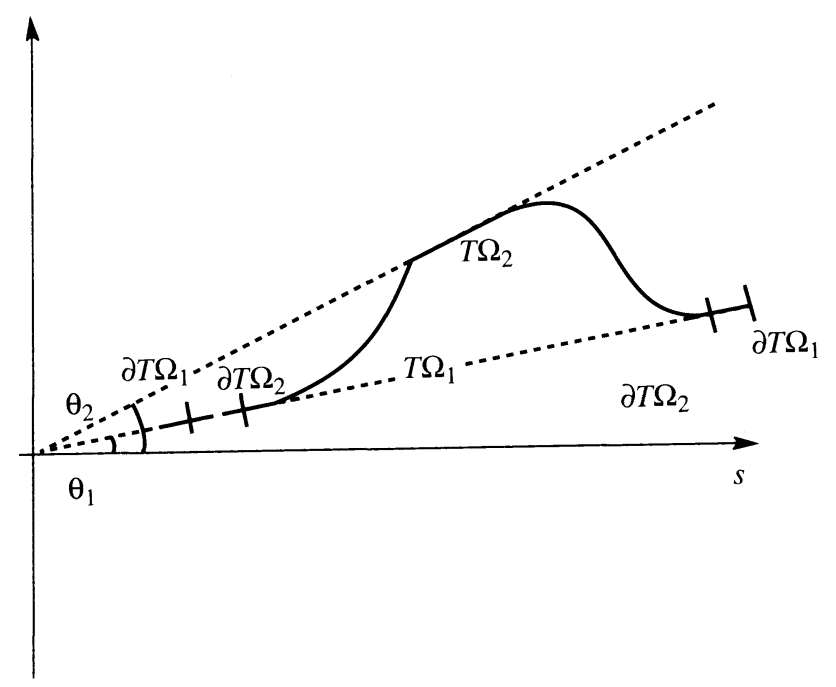

FIGURE 3.2 
are represented. Here the solid curve represents $\Gamma_{\theta_{1}, \theta_{2}, T}$ and $T \Omega_{1}$ and $T \Omega_{2}$ are indicated as subsets of that curve. If $u_{\theta_{1}} \in \mathscr{D}$ and $\left(P_{\theta_{1}}-z_{0}\right) u_{\theta_{1}}=0$ then $u$ and its derivatives up to order 2 are $\mathscr{O}\left(e^{-k|x|}\right)$ near infinity, provided that $z_{0} e^{2 i \theta}$ is outside the closed parabolic neighbourhood of $[0,+\infty$ [ given by $\Re z \geq-k^{2},|\Im z| \leq 2 R\left((\Re z)^{2}+R^{2}\right)^{1 / 2}$. Using (3.19) with $-e^{-2 i \theta} \Delta_{y}$ replaced by the restriction of $-\Delta$ to $\Gamma_{\theta_{1}, \theta_{2}, T}$ and varying $T$, we see that $u_{\theta_{2}}$ is also $\mathscr{D}_{\theta_{2}}$ when $\theta_{2}-\theta_{1}$ is small enough. Iterating this argument finitely many times, we get the lemma.

Combining Lemmas 3.2 and 3.3 with analytic Fredholm theory (as developed for instance in the appendix of [6]) we see that the spectrum of $P_{\theta}$ in $\mathbb{C} \backslash e^{-2 i \theta}[0,+\infty[$ is a discrete set. (In particular, the spectrum of $P$ in $]-\infty, 0[$ is purely discrete.) If $\theta<\pi / 2$, this discrete set consists of the negative eigenvalues of $P$ plus a discrete set of values in the angle $0 \leq \arg z<2 \theta$. If $\pi / 2 \leq \theta<\pi$ then the spectrum of $P_{\theta}$ in $\mathbb{C} \backslash e^{-2 i \theta}[0,+\infty[$ is contained in $0 \leq \arg z<2 \theta$ and Lemma 3.4 tells us that the elements of this set can be defined independently of $\theta$ in the following sense: We say that $z \in \mathbb{C} \backslash\{0\}$ with $0 \leq-\arg z<2 \pi$ is a resonance if and only if $z \in \sigma\left(P_{\theta}\right)$ for some $\pi \in[0, \pi[$ with $-\arg z<2 \theta$. This property is independent of the choice of $\theta$.

Applying the appendix of [6] we also see that if $z_{0}$ is a resonance and if $-\arg z<2 \theta<2 \pi$ then the spectral projection

$$
\pi_{\theta, z_{0}}=(2 \pi)^{-1} \int_{\gamma}\left(z-P_{\theta}\right)^{-1} d z, \quad \text { with } \gamma:\left[0,2 \pi\left[\ni \sigma \mapsto z_{0}+\varepsilon e^{i \sigma}\right.\right.
$$

and $\varepsilon>0$ small enough, is of finite rank. The image $F_{\theta, z_{0}}$ is contained in the domain of any power of $P_{\theta}$ and is invariant under $P_{\theta}$. Moreover, the restriction of $\left(P_{\theta}-z_{0}\right)$ to $F_{\theta, z_{0}}$ is nilpotent. The sequence $k \mapsto \operatorname{ker}\left(P_{\theta}-z_{0}\right)^{k}$ is increasing and stationary for $k \geq k_{0}$ for some finite $k_{0}$. We have $F_{\theta, z_{0}}=\operatorname{ker}\left(P_{\theta}-z_{0}\right)^{k_{0}}$. If $\tilde{\theta}$ is a second number with $-\arg z<2 \tilde{\theta}<2 \pi$, then Lemma 3.4 and its proof show that $F_{\theta, z_{0}}$ and $F_{\tilde{\theta}, z_{0}}$ are equal in the sense that holomorphic extension from $\Gamma_{\theta}$ followed by restriction to $\Gamma_{\tilde{\theta}}$ gives a bijection $F_{\theta, z_{0}} \rightarrow F_{\tilde{\theta}, z_{0}}$. The dimension of $F_{\theta, z_{0}}$ will be called the multiplicity of the resonance $z_{0}$.

To finish our discussion, we shall show that the resonances can also be described as the poles of the meromorphic extension of the resolvent $\left(z-P_{0}\right)^{-1}$ viewed as an operator $\mathscr{H}_{\text {comp }} \rightarrow \mathscr{D}_{\text {loc }}$. Here we define $\mathscr{H}_{\text {comp }}$ to be the space of elements in $\mathscr{H}$ that vanish outside a compact set, and $\mathscr{D}_{\text {loc }}$ is defined to be the space of elements $u$ in $\mathscr{H}_{R_{0}} \oplus H_{\mathrm{loc}}^{2}\left(\mathbb{R}^{n} \backslash B\left(0, R_{0}\right)\right)$ with the property that $\psi u \in \mathscr{D}$ for all $\psi \in C_{0}^{\infty}\left(\mathbb{R}^{n}\right)$ that are constant in a neighbourhood of $\overline{B\left(0, R_{0}\right)}$.

Lemma 3.5. The resolvent $(z-P)^{-1}: \mathscr{H}_{\mathrm{comp}} \rightarrow \mathscr{D}_{\mathrm{loc}}$ defined first for $\Im z>0$ can be extended holomorphically across $] 0,+\infty[$ to the complement in $\{z \in \mathbb{C} \backslash\{0\}$; $-\arg z \in[0,2 \pi[\}$ of the set of resonances. 
Proof. If $\Delta$ denotes the Laplacian on $\mathscr{R}^{n}$ then $(-\Delta-z)^{-1}: L_{\text {comp }}^{2}\left(\mathbb{R}^{n}\right) \rightarrow$ $H_{\text {loc }}^{2}\left(\mathbb{R}^{n}\right)$ has a holomorphic extension across $] 0,+\infty[$ to $\{z \in \mathbb{C} \backslash\{0\} ;-\arg z \in$ $\left[0,2 \pi[\}\right.$. We denote this extension by $R_{0}(z)$ (hence defiried for $-\pi<-\arg z<$ $2 \pi)$. For $-\pi<-\arg z<0$, we can write

$$
\begin{aligned}
(P-z)^{-1}= & (P-z)^{-1} \chi+(1-\tilde{\chi}) R_{0}(z)(1-\chi)(1-\chi), \\
& +(P-z)^{-1}[P, \tilde{\chi}] R_{0}(z)
\end{aligned}
$$

where $\tilde{\chi}, \chi \in C_{0}^{\infty}\left(B\left(0, R_{0}+a_{0}\right)\right)$ and $\chi=1$ near $\operatorname{supp} \tilde{\chi}, \tilde{\chi}=1$ near $\overline{B\left(0, R_{0}\right)}$. From this formula we see that it is enough to extend $(z-P)^{-1}: \mathscr{H}_{R_{0}+a_{0}}$ $\rightarrow \mathscr{D}_{\text {loc }}$, where $\mathscr{H}_{R_{0}+a_{0}}$ denotes the space of elements of $\mathscr{H}$ that vanish outside $B\left(0, R_{0}+a_{0}\right)$. If $v$ belongs to this space, we let $u_{\theta, z}$ denote the solution in $\mathscr{D}_{\theta}$ of $\left(P_{\theta}-z\right) u_{\theta, z}=v$, which exists, provided that $\max (-\pi, 2 \theta-2 \pi)<-\arg z<$ $2 \theta<2 \pi, \theta \geq 0$, and that $z$ is not a resonance. This solution depends holomorphically on $z$ and for $\max (-\pi, 2 \theta-2 \pi)<-\arg z<0$ it is the restriction to $\Gamma_{\theta}$ of the holomorphic extension of $(P-z)^{-1} v$ (outside the ball of radius $R_{0}+a_{0}$ ). For a fixed $\theta$, we get the required extension to $0 \leq-\arg z<2 \theta$ by taking the holomorphic extension of $u_{\theta, z}$ and restricting it to $\Gamma_{0}=\mathbb{R}^{n}$.

Let $z_{0}$ be a resonance. Then the deformation argument in the proof of Lemma 3.5 also shows that for $v \in \mathscr{H}_{R_{0}+a_{0}}$, we have

$$
(P-z)^{-1} v=\left(z_{0}-z\right)^{-N_{0}} v_{-N_{0}}+\cdots+\left(z_{0}-z\right)^{-1} v_{-1}+\operatorname{Hol}(z),
$$

for $z$ in a neighbourhood of $z_{0}$ and with $N_{0}$ independent of $v$. Here the $v_{-j}$ depend linearly on $v$. Put

$$
\pi_{0, z_{0}}=(2 \pi i)^{-1} \int(z-P)^{-1} d z: \mathscr{H}_{\text {comp }} \rightarrow \mathscr{D}_{\text {loc }},
$$

where we integrate the holomorphic extension along the same contour as in (3.20). If $v=v_{z} \in C_{0}^{\infty}\left(B\left(0, R_{0}+a_{0}\right) \backslash \overline{B\left(0, R_{0}\right)}\right)$ depends holomorphically on $z$ in a neighbourhood of $z_{0}$, then in the integral

$$
(2 \pi i)^{-1} \int(z-P)^{-1} v_{z} d z
$$

we may replace $v_{2}$ by a finite Taylor polynomial

$$
\sum_{0 \leq j \leq N_{0}}\left(z-z_{0}\right)^{j}\left(\partial_{z}^{j} v\right)_{z_{0}} / j ! .
$$

Since $\left(z-z_{0}\right)^{j}-\left(P-z_{0}\right)^{j}=(z-P)\left(\left(z-z_{0}\right)^{j-1}+\left(z-z_{0}\right)^{j-2}(z-P)+\cdots+\right.$ $\left.(z-P)^{j-1}\right)$, we can further replace $\left(z-z_{0}\right)^{j}$ by $\left(P-z_{0}\right)^{j}$. We then see that the integral (3.23) belongs to $\pi_{0, z_{0}}\left(C_{0}^{\infty}\left(B\left(0, R_{0}+a_{0}\right) \backslash \overline{B\left(0, R_{0}\right)}\right)\right)$. Using this observation and (3.21) we deduce that

$$
\pi_{0, z_{0}}\left(\mathscr{H}_{\text {comp }}\right)=\pi_{0, z_{0}}\left(\mathscr{H}_{R_{0}+a_{0}}\right) \text {. }
$$

By the deformation argument, we can identify the right-hand side of (3.25) with $\pi_{\theta, z_{0}}\left(\mathscr{H}_{R_{0}+a_{0}}\right)$ if $-\arg z_{0}<2 \theta<2 \pi$. We have then proved roughly one-half of the following result. 
Proposition 3.6. If $z_{0}$ is a resonance, then its multiplicity (defined above as the rank of the projection $\pi_{\theta, z_{0}}$ with $-\arg z_{0}<2 \theta<2 \pi$ ) is equal to the dimension of $\pi_{0, z_{0}}\left(\mathscr{H}_{\text {comp }}\right)$.

Proof. It only remains to show that

$$
\pi_{\theta, z_{0}}\left(\mathscr{H}_{R_{0}+a_{0}}\right)=\pi_{\theta, z_{0}}\left(\mathscr{H}_{\theta}\right) .
$$

We shall do this with a duality argument. Let $\Gamma_{-\theta}=\overline{\Gamma_{\theta}}$. We have a natural identification of $L^{2}\left(\Gamma_{\theta}\right)^{*}$ with $L^{2}\left(\Gamma_{-\theta}\right)$ given by

$$
\int_{\Gamma_{\theta}} u(x) \overline{v(\bar{x})} d x, \quad u \in L^{2}\left(\Gamma_{\theta}\right), v \in L^{2}\left(\Gamma_{-\theta}\right),
$$

where again $d x=\left.d x_{1} \wedge \cdots \wedge d x_{n}\right|_{\Gamma_{\theta}}$. From this we get a natural identification of $\mathscr{H}_{\theta}^{*}$ and $\mathscr{H}_{-\theta}$ given by a natural sesquilinear product $(u \mid v), u \in \mathscr{H}_{\theta}, v \in \mathscr{H}_{-\theta}$. Furthermore,

$$
\left(P_{\theta} u \mid v\right)=\left(u \mid P_{-\theta} v\right) \quad \text { when } u \in \mathscr{D}_{\theta}, v \in \mathscr{D}_{-\theta} .
$$

It follows that $z \notin \sigma\left(P_{\theta}\right)$ if and only if $\bar{z} \notin \sigma\left(P_{-\theta}\right)$ and that $\left(\left(P_{\theta}-z\right)^{-1}\right)^{*}=$ $\left(P_{-\theta}-\bar{z}\right)^{-1}$ when $z \notin \sigma\left(P_{\theta}\right)$. Now, $P_{\theta}^{*}=P_{-\theta}$, and hence $\left(\left(P_{\theta}-z\right)^{-1}\right)^{*}=$ $\left(P_{-\theta}-\bar{z}\right)^{-1}$, so that $\pi_{\theta, z_{0}}^{*}=\pi_{-\theta, \bar{z}_{0}}$, and in particular $\pi_{\theta, z_{0}}$ and $\pi_{-\theta, \bar{z}_{0}}$ have the same rank. It is also easy to prove that

$$
\pi_{\theta, z_{0}}^{2}=\pi_{\theta, z_{0}}
$$

If we write this as $\left(1-\pi_{\theta, z_{0}}\right) \pi_{\theta, z_{0}}=0$, we see that the images of $\pi_{\theta, z_{0}}$ and $1-\pi_{-\theta, \bar{z}_{0}}$ are orthogonal to each other and we can therefore identify the image of $\pi_{-\theta, \bar{z}_{0}}$ with the dual space of the image of $\pi_{\theta, z_{0}}$.

We can now prove (3.26). Assume that this identity does not hold. Then there exists $0 \neq u \in \pi_{-\theta, \bar{z}_{0}}\left(\mathscr{H}_{-\theta}\right)$ that is orthogonal to $\pi_{\theta, z_{0}}\left(\mathscr{H}_{R_{0}+a_{0}}\right)$. Using that $u=\pi_{-\theta, \bar{z}_{0}} u$, we get for all $v \in \mathscr{H}_{R_{0}+a_{0}}$

$$
0=\left(\pi_{\theta, z_{0}} v \mid u\right)=\left(v \mid \pi_{-\theta, \bar{z}_{0}} u\right)=(v \mid u),
$$

and hence $u(x)$ vanishes on $B\left(0, R_{0}+a_{0}\right)$. Since $u$ satisfies $\left(P_{-\theta}-\bar{z}_{0}\right)^{k} u=$ 0 , for some $k \in \mathbb{N}$ the holomorphic extension theorem, established in the proof of Lemma 3.1, implies that $u$ extends to a complex neighbourhood of $\Gamma_{-\theta} \backslash \overline{B\left(0, R_{0}\right)}$ and the vanishing on $B\left(0, R_{0}+a_{0}\right)$ then implies that $u$ vanishes completely in contradiction with the assumption that $u \neq \equiv 0$. This completes the proof of the lemma.

We shall now prove Theorem 1.1 in which we assume that $n$ is odd. The proof is essentially well known (cf. [25]), and we include it for the sake of completeness.

The free resolvent in odd dimensions is entire in the square root of the spectral parameter and thus, as in Lemma 3.5, we only need to prove the meromorphic continuation of $\left(P-\lambda^{2}\right)^{-1}: \mathscr{H}_{R_{0}+a_{0}} \rightarrow \mathscr{D}_{\text {loc }}$. Let us denote the free 
resolvent $\left(-\Delta-\lambda^{2}\right)^{-1}$, bounded on $L^{2}\left(\mathbb{R}^{n}\right)$ for $\lambda \in \mathbb{C}_{+}$, by $R_{0}(\lambda)$. The perturbed resolvent $\left(P-\lambda^{2}\right)^{-1}$, defined initially for $\lambda \in \mathbb{C}_{+}, \lambda^{2} \notin \sigma_{\text {point }}(P)$, is similarly denoted by $R(\lambda)$. If $\chi \in C_{0}^{\infty}\left(\mathbb{R}^{n}\right)$ and $\chi \equiv 1$ near $B\left(0, R_{0}+a_{0}\right)$, we want the meromorphy of $R(\lambda) \chi$.

Let us consider the following cut-off functions $\chi_{i} \in C_{0}^{\infty}\left(\mathbb{R}^{n}\right), i=0,1,2$, $\chi_{0} \equiv 1$ near $\overline{B\left(0, R_{0}\right)}$, and $\chi_{i} \equiv 1$ near $\operatorname{supp} \chi_{i-1}$. We then define the operators

$$
Q_{0}=\left(1-\chi_{0}\right) R_{0}(\lambda)\left(1-\chi_{1}\right), \quad Q_{1}=\chi_{2} R(\mu) \chi_{1},
$$

where $\mu \in \mathbb{C}_{+}$will be chosen later. Applying the operator $P-\lambda^{2}$ we obtain

$$
\left(P-\lambda^{2}\right) Q_{0}=I-\chi_{1}+\left[\Delta, \chi_{0}\right] R_{0}(\lambda)\left(1-\chi_{1}\right) \stackrel{\text { def }}{=} I-\chi_{1}+K_{0}(\lambda),
$$

and using the identity $P-\lambda^{2}=\left(P-\mu^{2}\right)-\left(\lambda^{2}-\mu^{2}\right)$,

$$
\left(P-\lambda^{2}\right) Q_{1}=\chi_{1}-\left[\Delta, \chi_{2}\right] R(\mu) \chi_{1}+\chi_{2}\left(\mu^{2}-\lambda^{2}\right) R(\mu) \chi_{1} \stackrel{\text { def }}{=} \chi_{1}+K_{1}(\lambda, \mu)
$$

If we take $\arg \mu=\pi / 4$ then in view of $(1.6) K_{1}(\lambda, \mu)$ and $K_{0}(\lambda) \chi$ are compact as operators $\mathscr{H} \rightarrow \mathscr{H}$ and entire in $\lambda$. Combining (3.31) and (3.32) we have

$$
\left(P-\lambda^{2}\right)\left(Q_{0}+Q_{1}\right)=I+K_{0}(\lambda)+K_{1}(\lambda, \mu),
$$

and for $\lambda \in \mathbb{C}_{+}, \lambda^{2} \notin \sigma_{\text {point }}(P)$,

$$
R(\lambda) \chi\left(I+K_{0}(\lambda) \chi+K_{1}(\lambda, \mu) \chi\right)=Q_{0} \chi+Q_{1} \chi .
$$

Thus the meromorphy of $R(\lambda) \chi$ follows from the meromorphy of the inverse of $I+K_{0}(\lambda) \chi+K_{1}(\lambda, \mu)$, which in turn follows from the analytic Fredholm theory once we show the invertibility at some point $\lambda$. Since $P$ is selfadjoint, taking $\arg \mu=\pi / 4$ gives $\|R(\mu)\|_{\mathscr{L}(\mathscr{H}, \mathscr{H})}=\mathscr{O}\left(|\mu|^{-2}\right)$ and $\|R(\mu)\|_{\mathscr{L}\left(\mathscr{H}, \mathscr{D}^{1 / 2}\right)}=$ $\mathscr{O}\left(|\mu|^{-1}\right) .^{\dagger}$ We clearly have the same estimate for $R_{0}(\mu)$ and the invertibility at $\lambda=\mu,|\mu|$ large, easily follows.

\section{Functional calculus for $P_{\theta}$}

We shall first define analogues of Sobolev spaces that are convenient in our discussion. The operator $P_{0}=h^{2} P$ introduced in $\S 1$ is selfadjoint, and we define $\mathscr{D}^{\alpha}, \alpha \geq 0$, to be the domain of the operator $\left\langle P_{0}\right\rangle^{\alpha}$ (we use the standard notation $\left.\langle\xi\rangle=\left(1+|\xi|^{2}\right)^{1 / 2}\right)$ equipped with the norm

$$
\|u\|_{\mathscr{D}^{\alpha}}=\left\|\left(P_{0}+i\right)^{\alpha} u\right\|_{\mathscr{H}} .
$$

For $\alpha<0$ we define $\mathscr{D}^{\alpha}$ to be the dual of $\mathscr{D}^{-\alpha}$, and it is easy to see that this space can be identified with the completion of $\mathscr{H}$ with respect to the norm (4.1).

Note that our norm has the semiclassical parameter $h$ built in, and similarly we introduce the modified semiclassical Sobolev norm

$$
\|u\|_{H^{s}\left(\mathbb{R}^{n}\right)}=\left\|\langle h D\rangle^{s} u\right\|_{L^{2}\left(\mathbb{R}^{n}\right)} .
$$

\footnotetext{
${ }^{\dagger}$ Strictly speaking we are using here the spaces and estimates introduced in $\S 4$ below.
} 
Let $C_{b}^{\infty}\left(\mathbb{R}^{n}\right)$ be the space of $C^{\infty}$ functions on $\mathbb{R}^{n}$ that are bounded together with all their derivatives.

Proposition 4.1. For every $k \in \mathbb{R}$ and every $\chi \in C_{b}^{\infty}\left(\mathbb{R}^{n}\right)$ with support disjoint from $\overline{B\left(0, R_{0}\right)}$ the multiplication operator $\chi$ is bounded $\mathscr{D}^{k} \rightarrow H^{2 k}\left(\mathbb{R}^{n}\right)$ and $H^{2 k}\left(\mathbb{R}^{n}\right) \rightarrow \mathscr{D}^{k}$ with a norm that is bounded independently of $h$.

Proof. The statement is clear for $k=0$. The uniform boundedness of $\chi: H^{2 k}\left(\mathbb{R}^{n}\right) \rightarrow \mathscr{D}^{k}$ follows from our general assumptions when $k \in \mathbb{N}$. On the other hand, if $u \in \mathscr{D}^{k}, k \in \mathbb{N}$, and if we choose $R_{0}<R_{1}<R_{2}$, then since $\left(-h^{2} \Delta+i\right)^{k} u \in H^{0}\left(C B\left(0, R_{1}\right)\right)$ and since $u_{\left|\subset B\left(0, R_{1}\right)\right|}$ is in $L^{2}$, it is standard that $\left.u\right|_{C B\left(0, R_{2}\right)}$ belongs to $H^{2 k}$ and that we have the following a priori estimate,

$$
\|u\|_{H^{2 k}\left(\mathcal{C} B\left(0, R_{2}\right)\right)} \leq C\left(\left\|\left(-h^{2} \Delta+1\right)^{k} u\right\|_{H^{0}\left(\mathcal{C} B\left(0, R_{1}\right)\right)}+\|u\|_{H^{0}\left(\mathcal{C} B\left(0, R_{1}\right)\right)}\right),
$$

where the constant $C$ will depend on $R_{j}$ and $k$ but not on $h$. It follows that $\chi$ is uniformly bounded $\mathscr{D}^{k} \rightarrow H^{2 k}\left(\mathbb{R}^{n}\right)$.

We have thus proved the proposition in the case when $k \in \mathbb{N}$, and by duality we can extend the validity to the case $k \in \mathbb{Z}$. In order to get the full result we shall use a simple form of complex interpolation. Consider, for instance, the problem of showing that $\chi$ is bounded $H^{2 k}\left(\mathbb{R}^{n}\right) \rightarrow \mathscr{D}^{k}$. In other words, we have to show that the operator $\left\langle P_{0}\right\rangle^{k} \chi\left\langle h^{2} \Delta\right\rangle^{-k}$ is bounded (uniformly with respect to $h): L^{2} \rightarrow \mathscr{H}$. We already know it for $k \in \mathbb{Z}$, and we want to extend it to the case of intermediate values. Fix $k \in \mathbb{Z}$, and consider for $\alpha$ with $k \leq$ $\Re \alpha \leq k+1$, the operator $\left\langle P_{0}\right\rangle^{\alpha} \chi\left\langle h^{2} \Delta\right\rangle^{-\alpha}$ that is uniformly bounded $H^{2} \rightarrow \mathscr{H}$, both with respect to $\alpha$ and $h$. Since $\left\langle P_{0}\right\rangle^{i \beta}$ and $\left\langle h^{2} \Delta\right\rangle^{-i \beta}$ are unitary when $\beta$ is real, we also know that $\left\langle P_{0}\right\rangle^{\alpha} \chi\left\langle h^{2} \Delta\right\rangle^{-\alpha}: L^{2} \rightarrow \mathscr{H}$ is uniformly bounded when $\operatorname{Re} \alpha$ is equal to $k$ or $k+1$. For $u \in H^{2}$, we then get with a constant $C$ which is independent of $\alpha$,

$$
\left\|\left\langle P_{0}\right\rangle^{\alpha} \chi\left\langle h^{2} \Delta\right\rangle^{-\alpha} u\right\|_{\mathscr{H}} \leq C\|u\|_{L^{2}} \quad \text { when } \Re \alpha \in\{k, k+1\},
$$

and $\left\|\left\langle P_{0}\right\rangle^{\alpha} \chi\left\langle h^{2} \Delta\right\rangle^{-\alpha} u\right\|_{\mathscr{H}} \leq C\|u\|_{H^{2}}$ when $k \leq \Re \alpha \leq k+1$. By the maximum principle (and the holomorphic dependence of $\alpha$ ) we then obtain that the inequality (4.3) extends to the whole strip and in particular to the case when $\alpha \in[k, k+1]$.

We fix some $\theta$ in $] \pi / 2,3 \pi / 4]$, and we shall let $z$ vary in $V=V_{\theta}=\{z \in$ $\mathbb{C}:|z| \geq \delta, 2 \pi-2 \theta-\varepsilon \geq \arg z \geq \delta\}$. Here $\delta, \varepsilon>0$ may be arbitrarily small and depending on the value of $\varepsilon$ we have to choose $\Gamma_{\theta}$ (see $\S 3$ ) in such a way that the values of the principal symbol of $P_{\theta}$ avoid a conic neighbourhood of $V$.

We have the following standard a priori estimate: For all $r>0, k \in$ $\mathbb{R}, M, N>0$, there exist constants $C>0, h_{0}>0$, such that if $\Omega_{1} \subset$ $\Omega_{2} \subset \mathbb{R}^{n}$ are open sets not intersecting $\overline{B\left(0, R_{0}\right)}$ and with the property that 
$\operatorname{dist}\left(\Omega_{1}, \mathbb{R}^{n} \backslash \Omega_{2}\right) \geq r>0$, then for all $u \in H^{k-M}\left(\Omega_{2}\right)$ and $z \in V$ with $\left(P_{\theta}-z\right) u \in H^{k}\left(\Omega_{2}\right)$, we have $u \in H^{k+2}\left(\Omega_{1}\right)$ and

$$
\begin{gathered}
\|u\|_{k+2, \Omega_{1}}+\langle z\rangle^{1 / 2}\|u\|_{k+1, \Omega_{1}}+\langle z\rangle\|u\|_{k, \Omega_{1}} \\
\leq C\left(\left\|\left(P_{\theta}-z\right) u\right\|_{k, \Omega_{2}}+h^{N}\|u\|_{k-M, \Omega_{2}}\right)
\end{gathered}
$$

for all $u \in H^{k+2}\left(\Omega_{2}\right)$ and for all $z \in V$ and all $\left.\left.h \in\right] 0, h_{0}\right]$. Here $\|u\|_{k, \Omega}$ denotes the norm of $u$ in $H^{k}(\Omega)$. Let $\chi \in C_{0}^{\infty}\left(B\left(0, R_{1}\right)\right)$ be equal to 1 near $\overline{B\left(0, R_{0}\right)}$. Then since $P_{0}$ is selfadjoint,

$$
\begin{aligned}
\|\chi u\|_{\mathscr{D}^{1}}+\langle z\rangle\|\chi u\| & \leq\left\|\left(P_{0}-z\right) \chi u\right\| \leq C\left(\left\|\chi\left(P_{\theta}-z\right) u\right\|+\left\|\left[P_{\theta}, \chi\right] u\right\|\right) \\
& \leq \widetilde{C}\left(\left\|\left(P_{\theta}-z\right) u\right\|+C h\|u\|_{H^{1}\left(\Omega_{1}\right)}\right),
\end{aligned}
$$

where $\Omega_{1} \subset \mathbb{R}^{n} \backslash \overline{B\left(0, R_{0}\right)}$ is any neighbourhood of $\operatorname{supp}(\nabla \chi)$. Choosing $k=$ $0, N=1, M=0$ in (4.4), and combining (4.4) and (4.5), we can get rid of the last term in the two estimates provided that $h>0$ is small enough, and we get that for every $R_{1}>0$ there exists $C>0$ such that for $h>0$ small enough

$$
\|u\|_{\mathscr{D}^{1}}+\langle z\rangle^{1 / 2}\|u\|_{H^{1}\left(C B\left(0, R_{1}\right)\right)}+\langle z\rangle\|u\| \leq C\left\|\left(P_{\theta}-z\right) u\right\|
$$

for $u \in \mathscr{D}^{1}, z \in V$

From this we get similar estimates in the whole scale of spaces $\mathscr{D}^{k}$. Indeed, let $u \in \mathscr{D}^{2}$ and apply (4.6) to $\left(P_{0}+i\right) u$. Using that $\left\|\left[P_{\theta}, P_{0}\right] u\right\| \leq$ $C h\|u\|_{H^{3}\left(C B\left(0, R_{1}\right)\right)}$ for some $R_{1}>R_{0}$ and that $C h\|u\|_{H^{3}\left(C B\left(0, R_{1}\right)\right)} \leq \frac{1}{2}\|u\|_{\mathscr{D}^{2}}+$ $\widetilde{C} h\|u\|$, where the last term can be estimated by means of (4.6), we get with a new constant $C$,

$$
\|u\|_{\mathscr{D}^{2}}+\langle z\rangle^{1 / 2}\|u\|_{H^{3}\left(\mathcal{C} B\left(0, R_{1}\right)\right)}+\langle z\rangle\|u\|_{\mathscr{D}^{1}} \leq C\left\|\left(P_{\theta}-z\right) u\right\|_{\mathscr{D}^{1}},
$$

when $z \in V$ and $H>0$ is sufficiently small. Repeating the argument, we see that for every $k \in \mathbb{N}$ there is a constant $C_{k}>0$ such that for $h>0$ sufficiently small and for $u \in \mathscr{D}^{k+1}, z \in V$

$$
\|u\|_{\mathscr{D}^{k+1}}+\langle z\rangle\|u\|_{\mathscr{D}^{k}} \leq C_{k}\left\|\left(P_{\theta}-z\right) u\right\|_{\mathscr{D}^{k}},
$$

and by interpolation, we could also add a term $\langle z\rangle^{1 / 2}\|u\|_{\mathscr{D}^{k+1 / 2}}$ to the left-hand side. This means, since $P_{\theta}-z$ is of index zero, that $P_{\theta}-z: \mathscr{D}^{1} \rightarrow \mathscr{H}$ is bijective and that the inverse $\left(P_{\theta}-z\right)^{-1}: \mathscr{H} \rightarrow \mathscr{D}^{1}$ is uniformly bounded $\mathscr{D}^{k} \rightarrow \mathscr{D}^{k+1}$,

$$
\left\|\left(P_{\theta}-z\right)^{-1}\right\|_{\mathscr{L}\left(\mathscr{D}^{k}, \mathscr{D}^{k+1}\right)}+\langle z\rangle\left\|\left(P_{\theta}-z\right)^{-1}\right\|_{\mathscr{L}\left(\mathscr{D}^{k}, \mathscr{D}^{k}\right)} \leq C_{k}
$$

for $z \in V$ and $h>0$ small enough. By duality, this can be extended to the case $k \in \mathbb{Z}$, if we notice that $\left(P_{\theta}-z\right)^{-1 *}=\left(P_{-\theta}-\bar{z}\right)^{-1}$ and that (4.9) holds for the latter operator. Finally by interpolation, we can extend the validity of (4.9) and (4.8) to the case when $k \in \mathbb{R}$. 


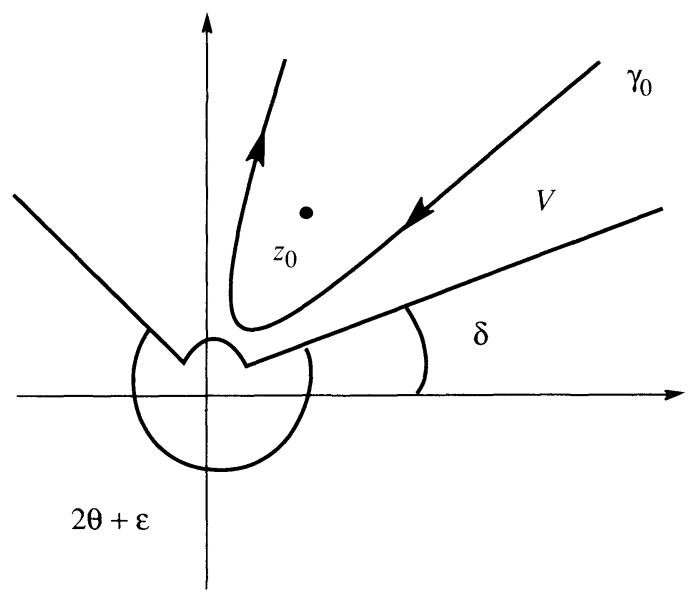

Figure 4.1

It is now straightforward to estimate negative integer powers of $\left(P_{\theta}-z\right)$ : For $k \in \mathbb{N} \backslash\{0\}$ and $l \in \mathbb{R}$ we get for sufficiently small $h>0$ and $z \in V$,

$$
\langle z\rangle^{k}\left\|\left(z-P_{\theta}\right)^{-k}\right\|_{\mathscr{L}\left(\mathscr{D}^{l}, \mathscr{D}^{l}\right)}+\cdots+\left\|\left(z-P_{\theta}\right)^{-k}\right\|_{\mathscr{L}\left(\mathscr{D}^{l}, \mathscr{D}^{l+k}\right)} \leq C_{k, l},
$$

where $C_{k, l}$ is independent of $h$ and $z$.

Let $\omega_{0} \in V$, and fix $\left.\delta_{0} \in\right] \delta, 2 \pi-2 \theta\left[\right.$ so that $\omega_{0}+e^{i \delta_{0}}\left[0,+\infty\left[\subset V^{\circ}\right.\right.$. Let $F$ be meromorphic in $\mathbb{C} \backslash e^{i \delta_{0}}\left[0,+\infty\left[\right.\right.$ with finitely many poles $z_{1}, \ldots, z_{N}$. (More generally we may take $F$ meromorphic and with finitely many poles in $\mathbb{C} \backslash \Gamma$, where $\Gamma$ is a closed set in $V^{\circ}$ that does not intersect some conic neighbourhood of $\partial V$.) We shall always assume that the poles of $F$ are outside the spectrum of $P_{\theta}$ and to start with we assume that there is an $\varepsilon>0$ and a constant $C>0$ such that

$$
|F(z)| \leq C|z|^{-\varepsilon} \quad \text { when }|z| \geq C
$$

We then put

$$
F\left(P_{\theta}\right)=(2 \pi i)^{-1} \int_{\gamma} F(z)\left(z-P_{\theta}\right)^{-1} d z,
$$

where $\gamma=\gamma_{0} \cup \gamma_{1} \cup \cdots \cup \gamma_{N}$. Here $\gamma_{0}$ is unbounded oriented from the right to the left and has the shape as in Figure 4.1.

The other curves $\gamma_{j}, j \geq 1$, are small circles centered at $z_{j}$ 's and oriented in the negative sense and containing no part of $\sigma\left(P_{\theta}\right)$ inside. It is also assumed that no poles of $F$ lie on $\gamma_{0}$ or between $\gamma_{0}$ and the cut. (The latter is not an essential restriction, if we have a number of poles between $\gamma_{0}$ and the cut, then simply suppress the corresponding $\gamma_{j}$ in the definition of $\gamma$. )

From the results of the preceding section, we see that $F\left(P_{\theta}\right)$ is a well-defined bounded operator $\mathscr{D}^{k} \rightarrow \mathscr{D}^{k}$ for every $k \in \mathbb{R}$ (but this may be valid only for $h>0$ sufficiently small depending on $k$ ). In fact, we only have to use that 
$\left\|\left(z-P_{\theta}\right)^{-1}\right\|_{\mathscr{L}\left(\mathscr{D}^{k}, \mathscr{D}^{k}\right)}=\mathscr{O}_{h}\left(\langle z\rangle^{-1}\right)$ for $z \in \gamma$, which follows in the case of $\gamma$ from $\S 1$ and which is also easy to establish (allowing then some $h$-dependence in the constant) for $z \in \gamma_{j}$.

Proposition 4.2. Let $G$ have the same properties as $F$. Then $F\left(P_{\theta}\right) G\left(P_{\theta}\right)=$ $(F G)\left(P_{\theta}\right)$.

Proof. By adding some small circles to the contours in the definitions of $F\left(P_{\theta}\right)$ and $G\left(P_{\theta}\right)$ we may first assume that these operators are defined as in (4.12) with the same contour $\gamma=\gamma_{0} \cup \gamma_{1} \cup \cdots \cup \gamma_{N}$. After that we "shrink" the contour in the definition of $G\left(P_{\theta}\right)$ into $\tilde{\gamma}=\tilde{\gamma}_{0} \cup \tilde{\gamma}_{1} \cup \cdots \cup \tilde{\gamma}_{N}$, where $\tilde{\gamma}_{0}$ lies above $\gamma_{0}$ and $\tilde{\gamma}_{j}$ lies inside $\gamma_{j}$ for $j \geq 1$. We then write

$$
\begin{aligned}
& F\left(P_{\theta}\right) G\left(P_{\theta}\right)=(2 \pi i)^{-2} \int_{\gamma} d z \int_{\tilde{\gamma}} d \tilde{z} F(z) G(\tilde{z})\left(z-P_{\theta}\right)^{-1}\left(\tilde{z}-P_{\theta}\right)^{-1} \\
& =(2 \pi i)^{-2} \int_{\gamma} d z \int_{\tilde{\gamma}} d \tilde{z} F(z) G(\tilde{z})(\tilde{z}-z)^{-1}\left[\left(z-P_{\theta}\right)^{-1}-\left(\tilde{z}-P_{\theta}\right)^{-1}\right] .
\end{aligned}
$$

Here we first look at

$$
\begin{aligned}
A & =(2 \pi i)^{-2} \int_{\gamma} d z \int_{\tilde{\gamma}} d \tilde{z} F(z) G(\tilde{z})(\tilde{z}-z)^{-1}\left(z-P_{\theta}\right)^{-1} \\
& =(2 \pi i)^{-1} \int_{\gamma} F(z)\left(z-P_{\theta}\right)^{-1}\left[(2 \pi i)^{-1} \int_{\tilde{\gamma}}(\tilde{z}-z)^{-1} G(\tilde{z}) d \tilde{z}\right] d z .
\end{aligned}
$$

For $z \in \gamma$, we have $(2 \pi i)^{-1} \int_{\tilde{\gamma}}(\tilde{z}-z)^{-1} G(\tilde{z}) d \tilde{z}=G(z)$, so we get $A=$ $(F G)\left(P_{\theta}\right)$. Then we look at

$$
\begin{aligned}
B & =(2 \pi i)^{-2} \int_{\gamma} d z \int_{\tilde{\gamma}} d \tilde{z} F(z) G(\tilde{z})(\tilde{z}-z)^{-1}\left(\tilde{z}-P_{\theta}\right)^{-1} \\
& =-(2 \pi i)^{-1} \int_{\tilde{\gamma}} G(\tilde{z})\left(\tilde{z}-P_{\theta}\right)^{-1}\left[(2 \pi i)^{-1} \int_{\gamma} F(z)(\tilde{z}-z)^{-1} d z\right] d \tilde{z} .
\end{aligned}
$$

Here $(2 \pi i)^{-1} \int_{\gamma} F(z)(\tilde{z}-z)^{-1} d z=0$ when $\tilde{z} \in \tilde{\gamma}$, so $B=0$.

If $F(z)=\mathscr{O}\left(|z|^{-1-\varepsilon}\right)$ near infinity for some $\varepsilon>0$, then we easily check that

$$
\left(P_{\theta}-\omega_{0}\right) F\left(P_{\theta}\right)=\left[\left(\cdot-\omega_{0}\right) F\right]\left(P_{\theta}\right) .
$$

We now relax the growth condition on $F$ to

$$
F(z)=\mathscr{O}\left(\langle z\rangle^{1-\varepsilon}\right)
$$

near infinity for some $\varepsilon>0$. Then if $w \in \mathbb{C} \backslash \sigma\left(P_{\theta}\right)$, we define

$$
F\left(P_{\theta}\right)=F_{w}\left(P_{\theta}\right)\left(P_{\theta}-w\right),
$$

where $F_{w}(z)=F(z) /(z-w)^{-1}$ satisfies the growth condition (4.11). $F\left(P_{\theta}\right)$ is then a bounded operator $\mathscr{D}^{k+1} \rightarrow \mathscr{D}^{k}$ for every $k \in \mathbb{R}$ and for every sufficiently small $h>0$. (If we want to bound the norm independently of $h$, however, we should assume that all the poles of $F$ are situated in the interior of $V$.) 
Naturally we have to check that the definition (4.15) is independent of the choice of $w$. We postpone this verification since we now pass to a more general case.

Assume that $F$ satisfies the growth condition

$$
F(z)=\mathscr{O}\left(|z|^{k-\varepsilon}\right)
$$

near infinity, where $k \in \mathbb{N}$ and where $\varepsilon>0$. We then define

$$
F\left(P_{\theta}\right)=F_{w}\left(P_{\theta}\right)\left(P_{\theta}-w_{1}\right) \circ \cdots \circ\left(P_{\theta}-w_{k}\right),
$$

where $w=\left(w_{1}, \ldots, w_{k}\right)$ avoids $\left(\sigma\left(P_{\theta}\right)\right)^{k}$ and

$$
F_{w}(z)=F(z)\left(z-w_{1}\right)^{-1} \cdots \cdot\left(z-w_{k}\right)^{-1} .
$$

For every $l \in \mathbb{R}$ this is a bounded operator $\mathscr{D}^{l+k} \rightarrow \mathscr{D}^{l}$ when $h>0$ is sufficiently small, and we have an $h$-independent bound for the norm when all the poles of $F$ lie inside $V^{\circ}$ and when we choose the $w_{j}$ in the same region. To check the $w$-independence, we differentiate with respect to $w_{1}$ (for instance) and get

$$
\begin{aligned}
\partial_{w_{1}} F\left(P_{\theta}\right)= & {\left[F_{w} /\left(\cdot-w_{1}\right)\right]\left(P_{\theta}\right)\left(P_{\theta}-w_{1}\right) \circ \cdots \circ\left(P_{\theta}-w_{k}\right) } \\
& -F_{w}\left(P_{\theta}\right)\left(P_{\theta}-w_{2}\right) \circ \cdots \circ\left(P_{\theta}-w_{k}\right),
\end{aligned}
$$

and if we use the identity (4.13), we see that the right-hand side vanishes. This shows that our definition is independent of $w$.

Proposition 4.3. Let $F$ satisfy the general assumptions above and assume in addition that

$$
F(z) \sim|z|^{\alpha} \quad \text { near infinity, }
$$

for some $\alpha \in] 0,1[$. Then

(A) If $w_{0} \in \mathbb{C} \backslash F\left(\sigma\left(P_{\theta}\right)\right)$ the operator $F\left(P_{\theta}\right)-w_{0}$ has a bounded two-sided inverse given by $\left[\left(F-w_{0}\right)^{-1}\right]\left(P_{\theta}\right)$.

(B) If $w_{0} \in F\left(\sigma\left(P_{\theta}\right)\right) \backslash F\left(e^{-2 i \theta}\left[0, \infty[)\right.\right.$ and if $F^{\prime}(z) \neq 0$ for every $z \in \sigma\left(P_{\theta}\right)$ with the property that $F(z)=w_{0}$, then we have

$$
\Pi_{F\left(P_{\theta}\right), w_{0}}=\sum_{j} \Pi_{P_{\theta}, z_{j}},
$$

where the $z_{j}$ are the finitely many points in $\sigma\left(P_{\theta}\right)$ with $F\left(z_{j}\right)=w_{0}$ and

$$
\Pi_{P_{\theta}, z_{j}}=(2 \pi i)^{-1} \int_{\gamma_{j}}\left(z-P_{\theta}\right)^{-1} d z
$$

is the spectral projection associated to $\left(P_{\theta}, z_{j}\right)$. Here $\gamma_{j}$ is a small positively oriented circle around $z_{j}$, and we define $\Pi_{F\left(P_{\theta}\right), z_{j}}$ analogously.

Proof. We start with the case (A). The function $G(z)=\left(F(z)-w_{0}\right)^{-1}$ is meromorphic outside $\omega_{0}+e^{+2 i \delta_{0}}[0,+\infty[$, is holomorphic outside a bounded set in $\mathbb{C} \backslash e^{+2 i \delta_{0}}\left[0,+\infty\left[\right.\right.$, and there satisfies: $|G(z)|=\mathscr{O}\left(\langle z\rangle^{-\alpha}\right)$. (Possibly there 
may be an infinite accumulation of poles towards the cut $e^{+2 i \delta_{0}}[0,+\infty[$, but as we have already remarked this is not an obstacle for defining $G\left(P_{\theta}\right)$.) Put $H(z)=F(z)-w_{0}$. For $w \notin \sigma\left(P_{\theta}\right)$ we get

$$
\begin{aligned}
H\left(P_{\theta}\right) G\left(P_{\theta}\right) & =H_{w}\left(P_{\theta}\right)\left(P_{\theta}-w\right) G\left(P_{\theta}\right)=H_{w}\left(P_{\theta}\right) G\left(P_{\theta}\right)\left(P_{\theta}-w\right) \\
& =\left[(\cdot-w)^{-1}\left(P_{\theta}\right)\right]\left(P_{\theta}-w\right)=1 .
\end{aligned}
$$

Here the last equality follows from the fact that

$$
(\cdot-w)^{-1}\left(P_{\theta}\right)=(2 \pi i)^{-1} \int_{\gamma}(z-w)^{-1}\left(z-P_{\theta}\right)^{-1} d z,
$$

and here we may (by deformation) assume that $\gamma$ is a small positively oriented circle around $w$. It is then clear that the right-hand side is equal to $-\left(w-P_{\theta}\right)^{-1}$. The proof of $(\mathrm{A})$ is complete since $G\left(P_{\theta}\right)$ and $H\left(P_{\theta}\right)$ commute.

We now consider

$$
w_{0} \in F\left(\sigma\left(P_{\theta}\right)\right) \backslash F\left(e^{-2 i \theta}[0, \infty[) .\right.
$$

If $\tilde{\gamma}$ is a small circle around $\omega_{0}$ we can write

$$
\begin{aligned}
\Pi_{F\left(P_{\theta}\right), w_{0}} & =\frac{1}{2 \pi i} \int_{\tilde{\gamma}}\left(z-F\left(P_{\theta}\right)\right)^{-1} d z \\
& =\frac{1}{2 \pi i} \int_{\tilde{\gamma}} d z \frac{1}{2 \pi i} \int_{\gamma^{\sharp}(z)}(z-F(t))^{-1}\left(t-P_{\theta}\right)^{-1} d t,
\end{aligned}
$$

where $\gamma^{\sharp}(z)=\gamma_{0} \cup \gamma_{1}(z) \cup \cdots \cup \gamma_{N}(z)$ with $\gamma_{0}$ the fixed contour defined in (4.12) and $\gamma_{j}(z)$ are small circles around the preimage $t_{j}(z)$ of $z, F\left(t_{j}(z)\right)=z$ (see Figure 4.2). The contour $\gamma_{j}(z)$ is homologous in $\mathbb{C} \backslash\left\{z_{j}\right\}$ to $\gamma_{j}^{i}+\gamma_{j}^{e}$ (see Figure 4.2) where $\gamma_{j}^{i}, \gamma_{j}^{e}$ are opposite oriented circles around $z_{j}$, independent of $z$

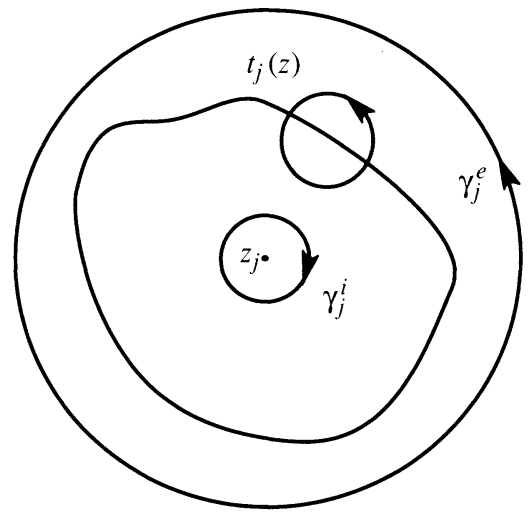

FIGURE 4.2 
and such that $\left\{t_{j}(z): z \in \tilde{\gamma}\right\}$ is sandwiched between them. Using this we can rewrite the right-hand side of (4.20) as

$$
\begin{aligned}
& \frac{1}{2 \pi i} \int_{\gamma_{0}}\left(\frac{1}{2 \pi i} \int_{\tilde{\gamma}}(z-F(t))^{-1} d z\right)(t-P)^{-1} d t \\
& \quad+\sum_{j=1}^{N} \frac{1}{2 \pi i} \int_{\gamma_{j}^{e}}\left(\frac{1}{2 \pi i} \int_{\tilde{\gamma}}(z-F(t))^{-1} d z\right)(t-P)^{-1} d t \\
& \quad+\sum_{j=1}^{N} \frac{1}{2 \pi i} \int_{\gamma_{j}^{i}}\left(\frac{1}{2 \pi i} \int_{\tilde{\gamma}}(z-F(t))^{-1} d z\right)(t-P)^{-1} d t
\end{aligned}
$$

The first two terms are easily seen to vanish as the $z$-integrals vanish (since $(z-F(t))^{-1}$ are holomorphic in the interior of $\tilde{\gamma}$ for $\left.t \in \gamma_{0}, \gamma_{j}^{e}\right)$, and we obtain

which is $(\mathrm{B})$ of the proposition.

$$
\Pi_{F\left(P_{\theta}\right), w_{0}}=\sum_{j=1}^{N}\left(\frac{1}{2 \pi} \int_{\gamma_{j}^{i}}\left(t-P_{\theta}\right)^{-1} d t\right)=\sum_{j=1}^{N} \Pi_{z_{j}, P},
$$

We now want to consider boundedness of the operator $F\left(P_{\theta}\right)$.

Proposition 4.4. Suppose that $F$ satisfies the general assumptions above and in addition

$$
F(z)=\mathscr{O}\left(\langle z\rangle^{\alpha}\right) \text { near infinity. }
$$

Then for every $\varepsilon>0$ and all $\alpha, \beta \in \mathbb{R}$ we have that

$$
F\left(P_{\theta}\right): \mathscr{D}^{\beta} \rightarrow \mathscr{D}^{\beta-\alpha-\varepsilon},
$$

provided that $0<h<h(\alpha, \beta)$ is small enough. If $F$ has no poles outside $V$, the norm of $F\left(P_{\theta}\right)$ is bounded by a constant, independent of $h$.

This is going to be an easy consequence of the definition of $F\left(P_{\theta}\right)$ and of the following

Lemma 4.5. For $z \in V, \alpha \in \mathbb{R}, \beta \in[0,1]$, and $0<h<h(\alpha, \beta)$ small enough,

$$
\left\|\left(z-P_{\theta}\right)^{-1}\right\|_{\mathscr{D}\left(\mathscr{D}^{\alpha}, \mathscr{D}^{\alpha+\beta}\right)} \leq \mathscr{O}_{\alpha}\left(\langle z\rangle^{\beta-1}\right) .
$$

Proof. Let us recall (4.8),

$$
\left(z-P_{\theta}\right)^{-1}= \begin{cases}\mathscr{O}_{\alpha}\left(\langle z\rangle^{-1}\right), & \mathscr{D}^{\alpha} \rightarrow \mathscr{D}^{\alpha}, \\ \mathscr{O}_{\alpha}(1), & \mathscr{D}^{\alpha} \rightarrow \mathscr{D}^{\alpha+1},\end{cases}
$$

from where we can proceed by complex interpolation. For that we define

$$
Q_{\beta}=\langle z\rangle^{1-\beta}\left\langle P_{0}\right\rangle^{\beta}\left\langle P_{0}\right\rangle^{\alpha}\left(z-P_{\theta}\right)^{-1}\left\langle P_{0}\right\rangle^{-\alpha},
$$

which is a holomorphic family of operators $\mathscr{D}^{\varepsilon} \rightarrow \mathscr{D}^{0}$ for every $\varepsilon>0$. Noting that $\left\langle P_{0}\right\rangle^{i \Im \beta}$ is unitary we infer that

$$
\left\|Q_{\beta}\right\|_{\mathscr{L}(\mathscr{H}, \mathscr{H})}=\mathscr{O}(\langle z\rangle) \text {. }
$$

On the other hand, (4.23) implies that

$$
\left\|Q_{\beta}\right\|_{\mathscr{L}(\mathscr{H}, \mathscr{H})}=\mathscr{O}(1) \text { for } \Re \beta=0 \text { or } \Re \beta=1 \text {. }
$$


An application of the Phragmén-Lindelöf principle to $Q_{\beta} u, u \in \mathscr{D}^{\varepsilon}$, gives

$$
\left\|Q_{\beta}\right\|_{\mathscr{L}(\mathscr{H}, \mathscr{L})}=\mathscr{O}(1), \quad 0 \leq \Re \beta \leq 1 .
$$

Recalling the definition of the $\mathscr{D}^{\gamma}$ norm, (4.1), we obtain the lemma.

To prove Proposition 4.4, let us assume first that $-1 \leq \alpha<0$. In view of the growth assumption and Lemma 4.5

$$
\left\|F(z)\left(z-P_{\theta}\right)^{-1}\right\|_{\mathscr{L}\left(\mathscr{D}^{\beta}, \mathscr{D}^{\beta-\alpha-\varepsilon}\right)}=\mathscr{O}\left(\langle z\rangle^{-1-\varepsilon}\right) .
$$

Thus, in the $\mathscr{L}\left(\mathscr{D}^{\beta}, \mathscr{D}^{\beta-\alpha-\varepsilon}\right)$, we obtain absolute convergence of the contour integrals which proves the statement.

For $\alpha \leq-1$ we consider

$$
F_{w}^{\sharp}(z)=F(z)\left(z-w_{1}\right) \cdots\left(z-w_{k}\right),
$$

$k=[-\alpha], \alpha \notin \mathbb{Z}, k=|\alpha|-1,|\alpha| \in \mathbb{Z}$, while for $\alpha \geq 0$

$$
F_{w}(z)=F(z)\left(z-w_{1}\right)^{-1} \cdots\left(z-w_{k}\right)^{-1}, \quad k=[\alpha]+1 .
$$

There we take $w_{i} \in V \backslash \sigma\left(P_{\theta}\right)$ so that $\left(P_{\theta}-w_{i}\right)^{-1}$ is an isomorphism of $\mathscr{H}$ and $\mathscr{D}$. Since the bound is already proved for $F_{w}^{\sharp}$ and $F_{w}$ above, the general case follows. To obtain the $h$-independence of the constant we need the assumption on the poles as in the comment following (4.18).

To obtain an improved bound for commutators of $F\left(P_{\theta}\right)$ with cutoff functions, let us first observe that if $\chi \in C_{b}^{\infty}\left(\mathbb{R}^{n}\right)$ and $\chi$ is constant in a neighbourhood of $\overline{B\left(0, R_{0}\right)}$, then

$$
\left\|\left[P_{\theta}, \chi\right]\right\|_{\mathscr{L}\left(\mathscr{D}^{\gamma}, \mathscr{D}^{\gamma-1 / 2}\right)}=\mathscr{O}_{\gamma}(h)
$$

for all $\gamma \in \mathbb{R}$. If $F$ satisfies the assumptions of Proposition 4.4 with $-1 \leq \alpha<$ 0 , then

$$
\left[F\left(P_{\theta}\right), \chi\right]=\frac{1}{2 \pi i} \int_{\gamma} F(z)\left(z-P_{\theta}\right)^{-1}\left[P_{\theta}, \chi\right] \cdot\left(z-P_{\theta}\right)^{-1} d z .
$$

The $\mathscr{L}\left(\mathscr{D}^{\beta}, \mathscr{D}^{\beta+1 / 2-\alpha-\varepsilon}\right)$ norm of the integrand is $\mathscr{O}_{\beta}\left(h\langle z\rangle^{-1-\varepsilon}\right)$ by Lemma 4.5 and (4.24) if we assume that the poles of $F$ are contained in $V$.

The case $\alpha \in \mathbb{R}$ is analyzed by modifying the argument in the proof of Proposition 4.4, and we obtain

Proposition 4.6. Suppose that $F$ satisfies the assumption of Proposition 4.4 and has no poles outside $V$. Then, for all $\varepsilon>0, \alpha, \beta \in \mathbb{R}$, and $\chi \in C_{b}^{\infty}(\mathbb{R})$ constant in a neighbourhood of $\overline{B\left(0, R_{0}\right)}$, we have that

$$
\left[F\left(P_{\theta}\right), \chi\right]=\mathscr{O}_{\alpha, \beta, \varepsilon}(h): \mathscr{D}^{\beta} \rightarrow \mathscr{D}^{\beta+1 / 2-\alpha-\varepsilon} .
$$

\section{SMOOTHING AND APPROXIMATION}

In this section we would like to localize the functional calculus of the previous section using the calculus of operators $P_{0}, \widetilde{P}_{\theta}$, and $\widehat{P}_{\theta}$,

$$
\widetilde{P}_{\theta}=-h^{2} \Delta_{\Gamma_{\theta}}, \quad \widehat{P}_{\theta}=-h^{2} e^{-2 i \theta} \Delta .
$$


Let us recall that $P_{\theta}$ is equal to $\widetilde{P}_{\theta}$ and $\widehat{P}_{\theta}$ in $\mathbb{R}^{n} \backslash \overline{B\left(0, R_{0}\right)}$ and $\mathbb{R}^{n} \backslash \overline{B\left(0, R_{1}\right)}$ respectively. Here $R_{1}>R_{0}$ is sufficiently large. The essential component in the localization is the off-diagonal smoothing. To describe it we introduce the following class of negligible operators:

$$
\begin{aligned}
\mathscr{R}^{\sharp}= & \{R: V \times] 0,1] \in(z, h) \mapsto R(z, h) \in \mathscr{L}(\mathscr{H}, \mathscr{H}): \\
& \quad \text { for every } \alpha, \beta \in \mathbb{R}, N \in \mathbb{N} \text { there exists } h(\alpha, \beta, N) \\
& \text { such that for } \left.0<h<h(\alpha, \beta, N), R(z, h)=\mathscr{O}\left(h^{N} /\langle z\rangle^{N}\right): \mathscr{D}^{\alpha} \rightarrow \mathscr{D}^{\beta}\right\} .
\end{aligned}
$$

We now have

Proposition 5.1. Let $\chi_{1}, \chi_{2} \in C_{b}^{\infty}\left(\mathbb{R}^{n}\right)$ be constant in a neighbourhood of $\overline{B\left(0, R_{0}\right)}$, and let

$$
\operatorname{dist}\left(\operatorname{supp} \chi_{1}, \operatorname{supp} \chi_{2}\right)>0 \text {. }
$$

Then

$$
\chi_{1}\left(z-P_{\theta}\right)^{-1} \chi_{2} \in \mathscr{R}^{\sharp} .
$$

Proof. For any $M \in \mathbb{N}$ we can find $\psi_{1}, \ldots, \psi_{M} \in C_{b}^{\infty}\left(\mathbb{R}^{n}\right)$ constant in a neighbourhood of $\overline{B\left(0, R_{0}\right)}$ and such that

$$
\chi_{1}=\chi_{1} \psi_{1}, \quad \psi_{i-1} \psi_{i}=\psi_{i-1}, \quad 1 \leq i \leq M, \quad \psi_{M} \chi_{2}=0 .
$$

Then

$$
\begin{aligned}
\chi_{1}\left(P_{\theta}-z\right)^{-1} \chi_{2}= & \chi_{1} \psi_{1} \cdots \psi_{M}\left(P_{\theta}-z\right)^{-1} \chi_{2} \\
= & \chi_{1}\left(P_{\theta}-z\right)^{-1}\left[P_{\theta}, \psi_{1}\right]\left(P_{\theta}-z\right)^{-1}\left[P_{\theta}, \psi_{2}\right] \\
& \cdot\left(P_{\theta}-z\right)^{-1} \cdots\left(P_{\theta}-z\right)^{-1}\left[P_{\theta}, \psi_{M}\right]\left(P_{\theta}-z\right)^{-1} \chi_{2} .
\end{aligned}
$$

Since $\left\|\left[P_{\theta}, \psi_{j}\right]\left(P_{\theta}-z\right)^{-1}\right\|_{\mathscr{L}\left(\mathscr{D}^{\gamma}, \mathscr{D}^{\gamma+1 / 4}\right)}=\mathscr{O}\left(h\langle z\rangle^{-1 / 4}\right)$ by Lemma 4.5 and (4.24), (5.1) implies that

$$
\chi_{1}\left(P_{\theta}-z\right)^{-1} \chi_{2}=\mathscr{O}\left(h^{M}\langle z\rangle^{-M / 4}\right): \mathscr{D}^{\gamma} \mapsto \mathscr{D}^{\gamma+M / 4} .
$$

Putting $\gamma=\alpha$ and $M \geq \max (N, 4(\alpha-\beta))$ we conclude the proof.

Clearly the statement holds for $\widetilde{P}_{\theta}$ and $\widehat{P}_{\theta}$ if we replace $\mathscr{D}^{\alpha}$ by $H^{2 \alpha}$.

We can now approximate the resolvent of $P_{\theta}, \widetilde{P}_{\theta}$, and $\widehat{P}_{\theta}$ in corresponding regions:

Proposition 5.2. Let $\left.r_{1}, r_{2} \in\right] R_{0}, \infty\left[, r_{1}<r_{2}\right.$, be such that for some $\delta>0$,

$$
P_{\theta} u=P_{0} u \quad \text { if } \operatorname{supp} u \subset B\left(0, r_{1}+\delta\right)
$$

and

$$
P_{\theta} u=\widehat{P}_{\theta} u \quad \text { if } \operatorname{supp} u \subset \complement B\left(0, r_{2}-\delta\right) .
$$

Then

(A) If $\chi \in C_{0}^{\infty}\left(B\left(0, r_{1}\right)\right)$ is constant in a neighbourhood of $\overline{B\left(0, R_{0}\right)}$ then

$$
\left(z-P_{\theta}\right)^{-1} \chi=\left(z-P_{0}\right)^{-1} \chi+R(z) \chi, \quad R \in \mathscr{R}^{\sharp} .
$$


(B) If $\chi \in C_{0}^{\infty}\left(\mathbb{R}^{n} \backslash \overline{B\left(0, r_{1}\right)}\right)$ and if $\psi \in C_{0}^{\infty}\left(\mathbb{R}^{n}\right)$ equal to 1 near $\overline{B\left(0, R_{0}\right)}$ satisfies

$$
\operatorname{supp} \chi \cap \operatorname{supp} \psi=\varnothing
$$

then

$$
\left(z-P_{\theta}\right)^{-1} \chi=(1-\psi)\left(z-\widetilde{P}_{\theta}\right)^{-1} \chi+R(z) \chi, \quad R \in \mathscr{R}^{\sharp} .
$$

(C) If $\chi \in C_{b}^{\infty}\left(\mathbb{R}^{n}\right)$ has its support in $\mathbb{R}^{n} \backslash \overline{B\left(0, r_{2}\right)}$ and $\psi \in C_{0}^{\infty}$ is the same as in (B) then

$$
\left(z-P_{\theta}\right)^{-1} \chi=(1-\psi)\left(z-\widehat{P}_{\theta}\right)^{-1} \chi+R(z) \chi, \quad R \in \mathscr{R}^{\sharp} .
$$

Proof. We start with (A): the choice of $r_{1}$ together with Proposition 5.1 shows that

$$
\left(z-P_{\theta}\right)\left(z-P_{0}\right)^{-1} \chi=\chi+\left(P_{0}-P_{\theta}\right)\left(z-P_{\theta}\right)^{-1} \chi=\chi+K(z) \chi, \quad K \in \mathscr{R}^{\sharp} .
$$

We then get (A) with $R(z)=-\left(z-P_{\theta}\right)^{-1} K(z)$. The proofs of (B) and (C) are identical and similar to that of $(\mathrm{A})$. Let us consider $(\mathrm{B})$ :

$$
\left(z-P_{\theta}\right)(1-\psi)\left(z-\widetilde{P}_{\theta}\right)^{-1} \chi=\chi+\left[P_{\theta}, \psi\right]\left(z-\widetilde{P}_{\theta}\right)^{-1} \chi=\chi+K(z) \chi,
$$

where again $K \in \mathscr{R}^{\sharp}$. Hence we get (B) with $R(z)=-\left(z-P_{\theta}\right)^{-1} K(z)$.

For the purposes of this section we shall specialize in our functional calculus to taking (real) powers of $P_{\theta}$. One could actually generalize what follows to more general $F$ considered in $\S 4$, and in $\S 6$ we shall consider $F$ 's of the form $\left(\left(z-\omega_{0}\right)^{1 / 3}-\omega_{1}\right)^{-1}$. Again we introduce a class of residual operators, now without the parameter $z$,

$\mathscr{R}=\{[0,1] \ni h \mapsto R(h) \in \mathscr{L}(\mathscr{H}, \mathscr{H}):$ for all $\alpha, \beta \in \mathbb{R}, x \in \mathbb{N}$,

$$
\left.0<h<h(\alpha, \beta, N), R(h)=\mathscr{O}\left(h^{N}\right): \mathscr{D}^{\alpha} \rightarrow \mathscr{D}^{\beta}\right\} .
$$

First we have the analogue of Proposition 5.1:

Proposition 5.3. Let $\chi_{1}, \chi_{2}$ be as in Proposition 5.1, and let $\gamma \in \mathbb{R}$. Then

$$
\chi_{1}\left(P_{\theta}-\omega_{0}\right)^{\gamma} \chi_{2} \in \mathscr{R} \text {. }
$$

Proof. This is trivial for $\gamma \in \mathbb{N}$ and follows easily from Proposition 5.1 when $\gamma<0$; we obtain an absolutely convergent integral with $\mathscr{O}\left(h^{N}\right)$ norm. The general case easily follows.

Similarly we have the analogue of Proposition 5.2:

Proposition 5.4. Let $\gamma \in \mathbb{R}, \omega_{0} \in V$, and $r_{1}, r_{2}$ be as in Proposition 5.2. If we choose $\chi$ and $\psi$ as in Proposition 5.2 in the respective cases (A), (B), (C), we obtain
(A) $\left(P_{\theta}-\omega_{0}\right)^{\gamma} \chi=\left(P_{\theta}-\omega_{0}\right)^{\gamma} \chi+R \chi, R \in \mathscr{R}$.
(B) $\left(P_{\theta}-\omega_{0}\right)^{\gamma} \chi=(1-\psi)\left(\widetilde{P}_{\theta}-\omega_{0}\right)^{\gamma} \chi+R \chi, R \in \mathscr{R}$.
(C) $\left(P_{\theta}-\omega_{0}\right)^{\gamma} \chi=(1-\psi)\left(\widehat{P}_{\theta}-\omega_{0}\right)^{\gamma} \chi+R \chi, R \in \mathscr{R}$. 
We now want to consider the trace class properties, and we first introduce the spaces of trace negligible operators corresponding to $\mathscr{R}^{\sharp}$ and $\mathscr{R}$. Thus we define

$\left.\mathscr{R}_{1}^{\sharp}=\{R: V \times] 0,1\right] \mapsto R(z, h) \in \mathscr{L}_{1}(\mathscr{H}, \mathscr{H}):$ for every $\alpha, \beta \in \mathbb{R}, N \in \mathbb{N}$,

$$
\text { and } \left.0<h<h(\alpha, \beta, N),\|R(z, h)\|_{\mathscr{L}_{1}\left(\mathscr{D}^{\alpha}, \mathscr{D}^{\beta}\right)}=\mathscr{O}\left(h^{N}\langle z\rangle^{-N}\right)\right\}
$$

and

$$
\begin{aligned}
\left.\mathscr{R}_{1}=\{R:] 0,1\right] & \rightarrow R(h) \in \mathscr{L}_{1}(\mathscr{H}, \mathscr{H}): \text { for every } \alpha, \beta \in \mathbb{R}, N \in \mathbb{N}, \\
& \text { and } \left.0<h<h(\alpha, \beta, N),\|R(h)\|_{\mathscr{L}_{1}\left(\mathscr{D}^{\alpha}, \mathscr{D}^{\beta}\right)}=\mathscr{O}\left(h^{N}\right)\right\} .
\end{aligned}
$$

Here $\mathscr{L}_{1}(A, B)$ denotes the space of trace class operators from $A$ to $B$.

Lemma 5.5. Suppose $R \in \mathscr{R}^{\sharp}($ or $R \in \mathscr{R})$ and $\chi \in C_{0}^{\infty}\left(\mathbb{R}^{n} \backslash \overline{B\left(0, R_{0}\right)}\right)$. Then

$$
\chi R, R \chi \in \mathscr{R}_{1}^{\sharp} \quad\left(\text { or } \mathscr{R}_{1}\right) \text {. }
$$

Proof. For $R \in \mathscr{R}$ we only need to consider $R \chi$ since $\mathscr{R}_{1}$ is closed under taking adjoints. Let us take $\chi, \chi_{1} \in C_{0}^{\infty}\left(\mathbb{R}^{n} \backslash \overline{B\left(0, R_{0}\right)}\right), \chi_{1} \chi=\chi$, and write

$$
R \chi=R \chi_{1}\left\langle-h^{2} \Delta\right\rangle^{N}\left\langle-h^{2} \Delta\right\rangle^{-N} \chi
$$

Then, if $N$ is sufficiently large,

$$
\chi_{1}\left\langle-h^{2} \Delta\right\rangle^{N}: H^{\alpha}\left(\mathbb{R}^{n}\right) \rightarrow \mathscr{D}^{\alpha-N}, \quad\left\|\left\langle-h^{2} \Delta\right\rangle^{-N} \chi\right\|_{\mathscr{L}_{1}\left(\mathscr{D}^{\beta}, H^{\alpha}\left(\mathbb{R}^{n}\right)\right)} \leq C h^{-N}
$$

so that

$$
\begin{aligned}
\|R \chi\|_{\mathscr{L}_{1}\left(\mathscr{D}^{\beta}, \mathscr{D}^{\alpha}\right)} & \leq C h^{-N}\|R\|_{\mathscr{L}\left(\mathscr{D}^{\alpha-N}, \mathscr{D}^{\alpha}\right)}\left\|\chi_{1}\left\langle-h^{2} \Delta\right\rangle^{N}\right\|_{\mathscr{L}\left(H^{\alpha}\left(\mathbb{R}^{n}\right), \mathscr{D}^{\alpha-N}\right)} \\
& =\mathscr{O}\left(h^{M-N}\right)
\end{aligned}
$$

i.e., $R \chi \in \mathscr{R}_{1}$, since $M$ is arbitrary.

We now see that the error terms in Propositions 5.2, 5.4 are actually trace negligible.

Proposition 5.6. (a) With the assumptions of Propositions 5.1 and 5.3,

$$
\chi_{1}\left(z-P_{\theta}\right)^{-1} \chi_{2} \in \mathscr{R}_{1}^{\sharp}, \quad \chi_{1}\left(P_{\theta}-\omega_{0}\right)^{\gamma} \chi_{2} \in \mathscr{R}_{1},
$$

respectively, if either $\chi_{1}$ or $\chi_{2}$ has compact support.

(b) The operators $R \chi$ in (A)-(C) of Propositions 5.2 and 5.4 are in $\mathscr{R}_{1}^{\sharp}$ and $\mathscr{R}_{1}$, respectively.

Proof. (a) Consider the case of Proposition 5.1, and suppose, say, that $\chi_{1}$ has compact support. If

$$
\operatorname{supp} \chi_{1} \cap \overline{B\left(0, R_{0}\right)}=\varnothing
$$

we are in the case of Lemma 5.5. Otherwise, take $M=1$ in (5.1) and $\psi_{M} \in$ $C_{0}^{\infty}\left(\mathbb{R}^{n}\right)$,

$$
\chi_{1}\left(P_{\theta}-z\right)^{-1} \chi_{2}=\chi_{1}\left(P_{\theta}-z\right)^{-1}\left[P_{\theta}, \psi_{1}\right]\left(P_{\theta}-z\right)^{-1} \chi_{2}
$$


Since $\left[P_{\theta}, \psi_{1}\right]$ has support disjoint from $\overline{B\left(0, R_{0}\right)}, \chi_{1}\left(P_{\theta}-z\right)^{-1} \chi_{2} \in \mathscr{R}_{1}^{\sharp}$. For the analogue of Proposition 5.3 we use the same argument 'inside' of the contour integral for $\gamma<0$. For general $\gamma$, we compare trace negligible operators $\left(\mathscr{R}_{1}\right)$ with possible integral powers of $\left(P_{\theta}-\omega\right)$.

(b) Since $\left\|\left(z-P_{\theta}\right)^{-1}\right\|_{\mathscr{L}\left(\mathscr{D}^{\alpha}, \mathscr{D}^{\alpha}\right)}=\mathscr{O}\left(\langle z\rangle^{-1}\right)$ we only need to show that the operators $K \chi$ in the proof of $(\mathrm{A})-(\mathrm{C})$ of Proposition 5.2 are in $\mathscr{R}_{1}^{\sharp}$. This follows, however, from the modification of (a) for $\widetilde{P}_{\theta}$. The case of Proposition 5.4 is now straightforward.

\section{6. $B_{\theta}$ AND ITS FUNCTIONAL CALCULUS}

We would like to understand the distribution of eigenvalues of the nonselfadjoint operator $\left(P_{\theta}-\omega_{0}\right)^{1 / 3}$, and we proceed by analyzing its characteristic values. Thus the selfadjoint operator that is the object of our study is

$$
B_{\theta}=Z_{\theta}^{*} Z_{\theta}, \quad Z_{\theta}=\left(P_{\theta}-\omega_{0}\right)^{1 / 3}-\omega_{1}, \quad \Im \omega_{1}>0 .
$$

Here we choose the branch of $z^{1 / 3}$ on $\mathbb{C} \backslash e^{i \delta}[0, \infty)$ that is positive on $(0, \infty)$.

As already mentioned in $\S 2$ we shall choose some fixed value of $\omega_{1}$ in our semiclassical analysis. See $\S 7$.

The operator $B_{\theta}$ is a positive symmetric operator, so we can take its Friedrichs extension (denoted also by $B_{\theta}$ ) with the domain

$$
\mathscr{D}^{2 / 3+\varepsilon} \subset \mathscr{D}\left(B_{\theta}\right) \subset \mathscr{D}^{2 / 3-\varepsilon} \text {. }
$$

We shall study the resolvent $\left(z-B_{\theta}\right)^{-1}$ for $z$ in some fixed bounded region, $\Omega$, of $\mathbb{C} \backslash \mathbb{R}$ with particular interest in the uniform behaviour as $z$ approaches the real axis.

We start with the standard estimate:

$$
|\Im z|\|u\| \leq\left\|\left(z-B_{\theta}\right) u\right\|, \quad u \in \mathscr{D}\left(B_{\theta}\right) .
$$

Since $Z_{\theta}^{-1}$ is of the form $F\left(P_{\theta}\right)$ with $F$ satisfying the assumption of Proposition 4.3 (with $\alpha=-1 / 3$ ), we obtain

$$
Z_{\theta}^{-1}, Z_{\theta}^{*-1}=\mathscr{O}(1): \mathscr{D}^{\alpha} \rightarrow \mathscr{D}^{\alpha+1 / 3-\varepsilon}
$$

for every $\alpha \in \mathbb{R}, \varepsilon>0$. It follows that

$$
B_{\theta}^{-1}=\mathscr{O}(1): \mathscr{D}^{\alpha} \rightarrow \mathscr{D}^{\alpha+2 / 3-\varepsilon} .
$$

Combined with (6.1) this gives

$$
|\Im z|\|u\|_{\mathscr{D}^{2 / 3-\varepsilon}} \leq C_{\varepsilon}\left\|\left(z-B_{\theta}\right) u\right\|
$$

for all $\varepsilon>0$. Defining $\check{B}_{\theta}=Z_{\theta} Z_{\theta}^{*}$ we get the same estimates for it and eventually

$$
|\Im z|\|u\|_{\mathscr{D}^{2 / 3+\alpha-\varepsilon}} \leq C_{\alpha, \varepsilon}\left\|\left(z-B_{\theta}\right) u\right\|_{\mathscr{D}^{\alpha+\varepsilon}}
$$


for all $\alpha \in \mathbb{R}$ and $\varepsilon>0$. In fact, we first obtain (6.3) for $\alpha \in \frac{1}{3} \mathbb{Z}$,

$$
\begin{aligned}
|\Im z|\|u\|_{\mathscr{D}^{1 / 3+2 / 3-\varepsilon}} & \leq C_{\varepsilon}|\Im z|\left\|Z_{\theta} u\right\|_{\mathscr{D}^{2 / 3-\varepsilon / 2}} \leq C_{\varepsilon}^{\prime}\left\|\left(z-\check{B}_{\theta}\right) Z_{\theta} u\right\| \\
& =C_{\varepsilon}^{\prime}\left\|Z_{\theta}\left(z-B_{\theta}\right) u\right\| \leq C_{\varepsilon, 1 / 3}\left\|\left(z-B_{\theta}\right)\right\|_{\mathscr{D}^{1 / 3+\varepsilon}}
\end{aligned}
$$

The general case follows by duality and interpolation. We can rewrite (6.3) as

$$
\left(z-B_{\theta}\right)^{-1}=\mathscr{O}_{\alpha, \varepsilon}\left(\frac{1}{|\Im z|}\right): \mathscr{D}^{\alpha+\varepsilon} \rightarrow \mathscr{D}^{\alpha+2 / 3-\varepsilon} .
$$

We now consider the analogue of Proposition 5.1. Note that now the complex parameter $z$ is not constrained to $V$, and we need to understand the uniform behaviour as $\Im z$ approaches 0 .

First we introduce classes of seminegligible and semitrace negligible operators analogous to $\mathscr{R}^{\sharp}$ and $\mathscr{R}_{1}^{\sharp}$,

$$
\begin{aligned}
\mathscr{R}^{b}= & \{R: \Omega \times] 0,1[\rightarrow \mathscr{L}(\mathscr{H}, \mathscr{H}): \text { for all } N \in \mathbb{N}, \alpha, \beta \in \mathbb{R} \\
& \text { there exists } M \in \mathbb{N} \text { such that } \\
& \left.\|R(z, h)\|_{\mathscr{L}\left(\mathscr{D}^{\alpha}, \mathscr{D}^{\beta}\right)}=\mathscr{O}_{\alpha, \beta}\left(h^{N}|\Im z|^{-M}\right) \text { for } 0<h \leq h(\alpha, \beta, N)\right\}
\end{aligned}
$$

and $\mathscr{R}_{1}^{b}$ in the analogous way.

We can now state

Proposition 6.1. If $\chi_{1}$ and $\chi_{2}$ are as in Proposition 5.1 then

$$
\chi_{1}\left(z-B_{\theta}\right)^{-1} \chi_{2} \in \mathscr{R}^{b} .
$$

If, in addition, $\chi_{1}$ or $\chi_{2}$ has compact support then

$$
\chi_{1}\left(z-B_{\theta}\right)^{-1} \chi_{2} \in \mathscr{R}_{1}^{b} \text {. }
$$

Proof. To obtain (6.4) we repeat the proof of Proposition 5.1:

$$
\begin{aligned}
\chi_{1}\left(z-B_{\theta}\right)^{-1} \chi_{2}= & (-1)^{N} \chi_{1}\left(z-B_{\theta}\right)^{-1}\left[B_{\theta}, \psi_{1}\right]\left(z-B_{\theta}\right)^{-1}\left[B_{\theta}, \psi_{2}\right] \\
& \cdots\left[B_{\theta}, \psi_{N}\right]\left(z-B_{\theta}\right)^{-1} \chi_{2} .
\end{aligned}
$$

We only need to observe that

$$
\left[B_{\theta}, \psi_{j}\right]=\mathscr{O}(h): \mathscr{D}^{\gamma} \rightarrow \mathscr{D}^{\gamma-2 / 3+1 / 2-\varepsilon}
$$

by Proposition 4.4 and use (6.3).

To obtain (6.5), we easily see that it holds if the compactly supported $\chi$ has support disjoint from $\overline{B\left(0, R_{0}\right)}$. Indeed, the proof of Lemma 5.5 is then applicable.

To consider the general case, let us assume that, say, $\chi_{1}$ has compact support. Using (6.6) with $N=1, \psi_{1} \chi_{1}=\chi_{1}, \psi_{1} \chi_{2}=0, \psi_{1} \in C_{0}^{\infty}\left(\mathbb{R}^{n}\right)$ we have

$$
\chi_{1}\left(z-B_{\theta}\right)^{-1} \chi_{2}=-\chi_{1}\left(z-B_{\theta}\right)^{-1}\left[B_{\theta}, \psi_{1}\right]\left(z-B_{\theta}\right)^{-1} \chi_{2} \text {. }
$$

We can now find $\chi \in C_{0}^{\infty}\left(\mathbb{R}^{n}\right)$ such that

$$
\operatorname{supp} \chi \cap \overline{B\left(0, R_{0}+\varepsilon\right)}=\varnothing, \quad(1-\chi) \cdot D \psi_{1}=0, \quad \chi \cdot \chi_{2}=0 .
$$


Inserting $1=1-\chi+\chi$ on the right side of the commutator we only need to analyze

$$
-\chi_{1}\left(z-B_{\theta}\right)^{-1}\left[B_{\theta}, \psi_{1}\right](1-\chi)\left(z-B_{\theta}\right)^{-1} \chi_{2},
$$

the other term being in $\mathscr{R}_{1}^{b}$. Now, however,

$$
\begin{aligned}
{\left[B_{\theta}, \psi_{1}\right](1-\chi)=} & Z_{\theta}\left[Z_{\theta}^{*}, \psi_{1}\right](1-\chi)+\left[Z_{\theta}, \psi_{1}\right] Z_{\theta}^{*}(1-\chi) \\
= & Z_{\theta}\left[Z_{\theta}^{*}, \psi_{1}\right](1-\chi)+\left[Z_{\theta}, \psi_{1}\right](1-\tilde{\chi}) Z_{\theta}^{*}(1-\chi) \\
& +\left[Z_{\theta}, \psi_{1}\right] \tilde{\chi} Z_{\theta}^{*}(1-\chi),
\end{aligned}
$$

where we choose $\tilde{\chi} \in C_{0}^{\infty}\left(\mathbb{R}^{n}\right)$ so that $\operatorname{supp} \tilde{\chi} \cap \overline{B\left(0, R_{0}+\varepsilon\right)}=\varnothing, \tilde{\chi} \chi=\tilde{\chi}$, $(1-\tilde{\chi}) \cdot D \psi_{1}=0$. Proposition 4.4 implies that

$$
\tilde{\chi} Z_{\theta}^{*}(1-\chi) \in \mathscr{R}_{1},
$$

while the definition of $Z_{\theta}$ together with Proposition 5.6(a) gives

$$
\left[Z_{\theta}^{*}, \psi_{1}\right](1-\chi),\left[Z_{\theta}, \psi_{1}\right](1-\tilde{\chi}) \in \mathscr{R}_{1} \text {. }
$$

Combining this with (6.3) concludes the proof.

We proceed to obtain the analogue of statements in $\S 5$.

Proposition 6.2. Let $r_{1}$ and $r_{2}$ be as in Proposition 5.2, and let $\chi$ and $\psi$ be chosen as in (A), (B), (C) of that proposition, respectively. Then, modulo $\mathscr{R}_{1}$

(A) $B_{\theta} \chi \equiv B_{0} \chi$,

(B) $B_{\theta} \chi \equiv(1-\psi) \widetilde{B}_{\theta} \chi$,

(C) $B_{\theta} \chi \equiv(1-\psi) \widehat{B}_{\theta} \chi$,

where $B_{0}, \widetilde{B}_{\theta}$, and $\widehat{B}_{\theta}$ are defined by replacing $P_{\theta}$ in $B_{\theta}$ by $P_{0}, \widetilde{P}_{\theta}$, and $\widehat{P}_{\theta}$, respectively.

Proof. Let us define $Z_{0}, \widetilde{Z}_{\theta}$, and $\widehat{Z}_{\theta}$ in the obvious way. Proceeding as in the second part of the proof of Proposition 6.1 we obtain (A), (B), (C). Indeed, in case $(\mathrm{A})$ we choose $\tilde{\chi} \in C_{0}^{\infty}\left(\mathbb{R}^{n}\right)$ supported in a small neighbourhood of the support of $\chi$ and satisfying $\tilde{\chi} \chi=\chi$. Then, modulo $\mathscr{R}_{1}$

$$
B_{\theta} \chi=Z_{\theta}^{*} Z_{\theta} \chi \equiv \tilde{\chi} Z_{\theta}^{*} Z_{\theta} \chi \equiv\left(\tilde{\chi} Z_{0}^{*}\right)\left(Z_{0} \chi\right) \equiv B_{0} \chi \text {. }
$$

In case $(\mathrm{C})$ we take $\tilde{\chi} \in C_{b}^{\infty}\left(\mathbb{R}^{n}\right)$ with $\operatorname{dist}(\operatorname{supp} \chi, \operatorname{supp}(1-\tilde{\chi}))>\varepsilon / 2$ for some small $\varepsilon>0$. Then modulo $\mathscr{R}_{1}$

$$
\begin{aligned}
B_{\theta} \chi & \equiv \tilde{\chi} Z_{\theta}^{*} Z_{\theta} \chi \equiv \tilde{\chi} \widehat{Z}_{\theta}^{*}(1-\psi)(1-\psi) \widehat{Z}_{\theta} \chi \\
& \equiv \tilde{\chi} \widehat{Z}_{\theta}^{*} \widehat{Z}_{\theta} \chi \equiv(1-\psi) \widehat{Z}_{\theta}^{*} \widehat{Z}_{\theta} \chi .
\end{aligned}
$$

The proof for case $(B)$ is the same. One should remark that strictly speaking we were manipulating trace negligible operators between $\mathscr{H}$ and $L^{2}$ rather than just elements of $\mathscr{R}_{1}$.

In the analogue of Proposition 5.6(a) $\mathscr{R}_{1}^{\sharp}$ is replaced by $\mathscr{R}_{1}^{b}$, the semi-trace negligible operator. 
Proposition 6.3. Let $\chi$ and $\psi$ be respectively the cut-off functions in the cases (A), (B), (C) of Proposition 5.2. Then in the corresponding cases we have, modulo $\mathscr{R}_{1}^{b}$

(A) $\left(B_{\theta}-z\right)^{-1} \chi \equiv\left(B_{0}-z\right)^{-1} \chi$,

(B) $\left(B_{\theta}-z\right)^{-1} \chi \equiv(1-\psi)\left(\widetilde{B}_{\theta}-z\right)^{-1} \chi$,

(C) $\left(B_{\theta}-z\right)^{-1} \chi \equiv(1-\psi)\left(\widehat{B}_{\theta}-z\right)^{-1} \chi$.

Proof. For the case (A), as before, we first consider

$$
\left(B_{\theta}-z\right)\left(B_{0}-z\right)^{-1} \chi=\chi+\left(B_{\theta}-B_{0}\right)\left(B_{0}-z\right)^{-1} \chi .
$$

Let $\tilde{\chi} \in C_{0}^{\infty}$ have support in a small neighbourhood of $\operatorname{supp} \chi$, and let $\tilde{\chi}=1$ near supp $\chi$. Then we write $\left(B_{\theta}-z\right)\left(B_{0}-z\right)^{-1} \chi=\chi+\left(B_{\theta}-B_{0}\right) \tilde{\chi}\left(B_{0}-z\right)^{-1} \chi+\left(B_{\theta}-B_{0}\right)(1-\tilde{\chi})\left(B_{0}-z\right)^{-1} \chi$.

The last term is in $\mathscr{R}_{1}^{b}$ by Proposition 6.1, and so is the second term, since by Proposition 6.2

$$
\left(B_{\theta}-B_{0}\right) \tilde{\chi} \in \mathscr{R}_{1} .
$$

Applying $\left(B_{\theta}-z\right)^{-1}$ to $(6.8)$ gives $(\mathrm{A})$.

In case $(\mathrm{C})$ we choose $\tilde{\psi} \in C_{0}^{\infty}\left(\mathbb{R}^{n}\right)$ equal to 1 near $\overline{B\left(0, R_{0}\right)}$ and with support in the interior of the region where $\psi=1$. Then modulo $\mathscr{R}_{1}^{b}$ $\left(B_{\theta}-z\right)(1-\psi)\left(\widehat{B}_{\theta}-z\right)^{-1} \chi \equiv(1-\tilde{\psi})\left(\widehat{B}_{\theta}-z\right)(1-\psi) \cdot\left(\widehat{B}_{\theta}-z\right)^{-1} \chi \equiv(1-\psi) \chi=\chi$. Again, the application of $\left(B_{\theta}-z\right)^{-1}$ gives $(C)$. The case (B) follows similarly.

Since our first goal is the study of the spectrum of $B_{\theta}$, we want to estimate $\operatorname{tr} f\left(B_{\theta}\right)$ where $f \in C_{0}^{\infty}$ with support containing the discrete spectrum. We therefore recall the formula $[8,16]$,

$$
f(A)=\frac{1}{2 \pi i} \int \partial_{\bar{z}} \tilde{f}(z)(z-A)^{-1} d z \wedge d \bar{z},
$$

where $A$ is any selfadjoint operator, $f \in C_{0}^{\infty}(\mathbb{R})$, and $\tilde{f} \in C_{0}^{\infty}(\mathbb{C})$ is an almost-analytic extension of $f$. This is very useful here since for $R \in \mathscr{R}_{1}^{b}$ and $\tilde{f}$ almost analytic we easily see that

$$
\int \partial_{\bar{z}} \tilde{f}(z) R(z) d z \wedge d \bar{z} \in \mathscr{R}_{1} .
$$

Let $r_{1}, r_{2}$ be as in Proposition 5.2. We choose a partition of unity

$$
1=\chi_{0}+\chi_{1}+\chi_{2}
$$

where $\chi_{0}, \chi_{1} \in C_{0}^{\infty}\left(\mathbb{R}^{n}\right), \chi_{2} \in C_{b}^{\infty}\left(\mathbb{R}^{n}\right)$ with properties

$$
\begin{gathered}
\operatorname{supp} \chi_{0} \subset B\left(0, r_{1}\right), \quad \operatorname{supp} \chi_{2} \subset \mathbb{R}^{n} \backslash \overline{B\left(0, r_{2}\right)}, \\
\operatorname{supp} \chi_{1} \subset B\left(0, r_{2}+\varepsilon\right) \backslash B\left(0, r_{1}-\varepsilon\right),
\end{gathered}
$$

with $\varepsilon>0$, small.

Proposition 6.3, combined with (6.9) and (6.10), implies 
Theorem 6.4. If $f \in C_{0}^{\infty}(\mathbb{R})$ and $\chi_{0}, \chi_{1}, \chi_{2}$ is the partition of unity above then

$$
f\left(B_{\theta}\right)=f\left(B_{0}\right) \chi_{0}+\left(1-\psi_{1}\right) f\left(\widetilde{B}_{\theta}\right) \chi_{1}+\left(1-\psi_{2}\right) f\left(\widehat{B}_{\theta}\right) \chi_{2}+R, \quad R \in \mathscr{R}_{1},
$$

where $\psi_{j} \in C_{0}^{\infty}\left(\mathbb{R}^{n}\right)$ are equal to 1 near $\overline{B\left(0, R_{0}\right)}$ and $\operatorname{supp} \psi_{j} \cap \operatorname{supp} \chi_{j}=\varnothing$.

\section{Proof of the MAIN ESTiMATE}

To estimate the number of scattering poles we can, thanks to results of $\S 3$, estimate the number of eigenvalues of $P_{\theta}$ in a conveniently chosen region. Because of the presence of the semiclassical parameter we may consider a fixed region

$$
\Omega_{0}=\{z \in \mathbb{C}: \Im z \leq 0, R \leq|z| \leq 4 R\},
$$

with $R$ large (see Figure 7.1). Let us recall from $\S 3$ that, because of symmetry of the poles with respect to imaginary axis the restriction to $\Im z \leq 0$ is immaterial (the poles correspond to square roots of eigenvalues of $P_{\theta}$ ).

The application of the map

$$
z \mapsto\left(z-\omega_{0}\right)^{1 / 3}, \quad \Im \omega_{0}>0
$$

gives a region $\Omega$, shown in Figure 7.2 on p. 764. Note that $\left(P_{\theta}-\omega_{0}\right)^{1 / 3}$ has only discrete spectrum away from $\sigma\left(\left(\widehat{P}_{\theta}-\omega_{0}\right)^{1 / 3}\right)$.

It is clear that we can find $\omega_{1} \in \mathbb{C}, \Im \omega_{1}>0$ such that

$$
\Omega \subset D\left(\omega_{1}, R_{1}\right), \quad \overline{D\left(\omega_{1}, R_{3}\right)} \cap \sigma_{\text {ess }}\left(\left(P_{\theta}-\omega_{0}\right)^{1 / 3}\right)=\varnothing,
$$

with suitable $0<R_{1}<R_{2}<R_{3}$ and where we use notation $D(\omega, r)=\{z \in$ $\mathbb{C}:|z-\omega|<r\}$. We shall estimate $M\left(R_{2}, h\right)$, the number of eigenvalues of

$$
\left[\left(\left(P_{\theta}-\omega_{0}\right)^{1 / 3}-\omega_{1}\right)^{*}\left(\left(P_{\theta}-\omega_{0}\right)^{1 / 3}-\omega_{1}\right)\right]^{1 / 2}
$$

less than $R_{2}$.

We first make the following observation.

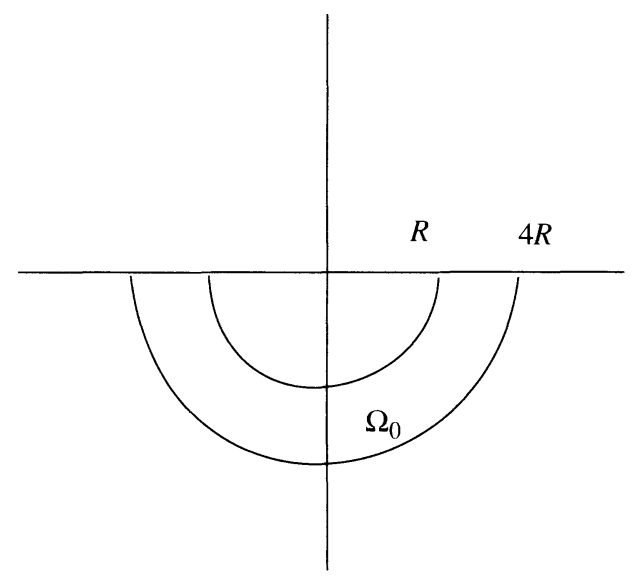

Figure 7.1 


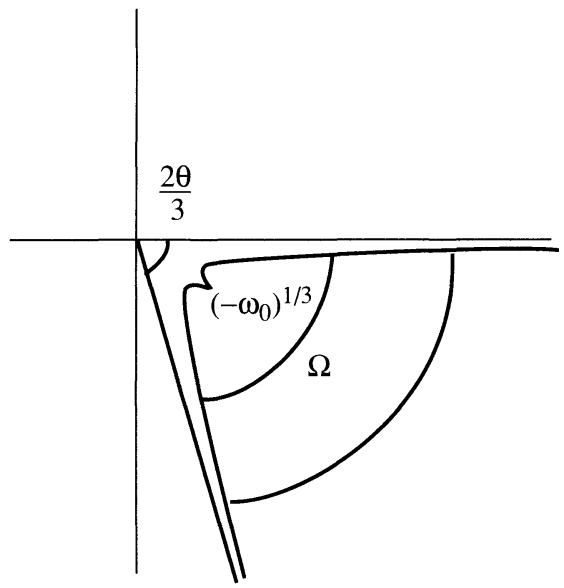

FIGURE 7.2

Lemma 7.1. There exists an $r>0$, independent of $h$, such that for $h$ sufficiently small

$$
\left\langle B_{\theta} u, u\right\rangle \geq r\|u\|^{2} .
$$

Proof. The inequality (7.2) is equivalent to $\left\|Z_{\theta} u\right\|^{2} \geq r\|u\|^{2}$. As in $\S 6$, this can be inferred from Proposition 4.4,

if $h$ is small enough.

$$
\left\|Z_{\theta}^{-1} u\right\| \leq\left\|Z_{\theta}^{-1} u\right\|_{\mathscr{D}^{1 / 3-\varepsilon}} \leq C\|u\|
$$

If we now take $f \in C_{0}^{\infty}(\mathbb{R},[0,+\infty))$ such that

then

$$
f(t)= \begin{cases}1, & 0 \leq t \leq R_{2}^{2}, \\ 0, & t \geq R_{3}^{2},\end{cases}
$$

$$
M\left(R_{2}, h\right) \leq \operatorname{tr} f\left(B_{\theta}\right) .
$$

Indeed, by Lemma 7.1, $B_{\theta}$ is bounded away from 0 . Also, the right-hand side of (7.3) is finite if $f\left(B_{0}\right) \chi_{0}, \chi_{0} \in C_{0}^{\infty}(\mathbb{R})$, is of trace class. To see this we use Theorem 6.4: $f\left(\widetilde{B}_{0}\right) \chi_{1}$ is of trace class by pseudodifferential functional calculus and $f\left(\widehat{B}_{\theta}\right)=0$ by $(7.1)$. In fact,

$$
\begin{aligned}
\widehat{B}_{\theta} & =\left(\left(\widehat{P}_{\theta}-\omega_{0}\right)^{1 / 3}-\omega_{1}\right)^{*}\left(\left(\widehat{P}_{\theta}-\omega_{0}\right)^{1 / 3}-\omega_{1}\right) \\
& =\left(\left(e^{-2 i \theta} \widehat{P}_{0}-\omega_{0}\right)^{1 / 3}-\omega_{1}\right)^{*}\left(\left(e^{-2 i \theta} \widehat{P}_{0}-\omega_{0}\right)^{1 / 3}-\omega_{1}\right) \\
& =\left(\left(e^{2 i \theta} \widehat{P}_{0}-\bar{\omega}_{0}\right)^{1 / 3}-\bar{\omega}_{1}\right)\left(\left(e^{-2 i \theta} \widehat{P}_{0}-\omega_{0}\right)^{1 / 3}-\omega_{1}\right) .
\end{aligned}
$$

Since $\sigma\left(\widehat{P}_{0}\right)=\overline{\mathbb{R}}_{+}$, the spectrum of $\widehat{B}_{\theta}$ is contained in

$$
\left.\left\{\left|\left(e^{-2 i \theta} t-\omega_{0}\right)^{1 / 3}-\omega_{1}\right|^{2}: t \geq 0\right\} \subset\right] R_{3}^{2}, \infty[.
$$

We now need to estimate $\operatorname{tr} f\left(\widetilde{B}_{\theta}\right) \chi_{1}$ and this is done in 
Lemma 7.2. With $\widetilde{B}_{\theta}$ and $\chi_{1}$ as in Theorem 6.1, we have

$$
\operatorname{tr} f\left(\widetilde{B}_{\theta}\right) \chi_{1}=\mathscr{O}\left(h^{-n}\right) \text {. }
$$

Proof. We will use the semiclassical pseudodifferential calculus, and first we need to review the construction of $\widetilde{B}_{\theta}$. It follows from $\S 2$ of [7] that for $z$ in a compact subset of $V$, and then for all $z \in V$ by rescaling we have $\left(z-\widetilde{P}_{\theta}\right)^{-1}=Q^{w}\left(x, h D_{x}, z, h\right)$, where the semiclassical symbol $Q(x, \xi, h, z)$ satisfies the estimates $\partial_{x}^{\alpha} \partial_{\xi}^{\beta} Q=\mathscr{O}\left(|z|^{-|\beta| / 2}\left(|z|+|\xi|^{2}\right)^{-2}\right)$ uniformly in $h$ (we are not concerned here with the symbolic behaviour in $\xi$ ). We also have an asymptotic expansion in $h$,

$$
\begin{aligned}
Q(x, \xi, z, h) & \sim Q_{0}(x, \xi, z)+h Q_{1}(x, \xi, z)+\cdots, \\
\partial_{x}^{\alpha} \partial_{\xi}^{\beta} Q_{j} & =\mathscr{O}\left(|z|^{-(|\beta|+j) / 2}\left(|z|+|\xi|^{2}\right)^{-1}\right) .
\end{aligned}
$$

If $\gamma_{0}$ is the contour in Figure 4.1 then we defined

$$
\left(\widetilde{P}_{\theta}-\omega_{0}\right)^{-2 / 3}=\frac{1}{2 \pi i} \int_{\gamma_{0}}\left(z-\widetilde{P}_{\theta}\right)^{-1}\left(z-\omega_{0}\right)^{-2 / 3} d z .
$$

This is again an $h$-pseudodifferential operator with the symbol obtained from $Q$ by the same integration formula. Since $\left(\widetilde{P}_{\theta}-\omega_{0}\right)^{1 / 3}=\left(\widetilde{P}_{\theta}-\omega_{0}\right)\left(\widetilde{P}_{\theta}-\omega_{0}\right)^{-2 / 3}$, the cubic root is also an $h$-pseudodifferential operator with the symbol in $S\left(\langle\xi\rangle^{2 / 3}\right)$. Finally we obtain that $\widetilde{B}_{\theta}$ is an $h$-pseudodifferential operator with the symbol in $S\left(\langle\xi\rangle^{4 / 3}\right.$ ) having a complete asymptotic expansion in $h$ with the leading term $\left|\left(\widetilde{P}_{\theta}-\omega_{0}\right)^{1 / 3}-\omega_{1}\right|^{2}$. For $(x, \xi)$ outside some bounded set in $\mathbb{R}^{2 n}$ this symbol takes its values above the support of $f$. Thus we can find $\chi \in C_{0}^{\infty}\left(\mathbb{R}^{2 n}\right)$ such that $\chi+\left|\left(\widetilde{P}_{\theta}-\omega_{0}\right)^{1 / 3}-\omega_{1}\right|^{2}$ takes its values above the support of $f$. Thus we define $B_{\theta}^{\natural}=\widetilde{B}_{\theta}+\chi^{w}\left(x, h D_{x}\right)$. Recalling (6.9), we may arrange that for $z$ in a neighbourhood of the support of $f$, and when $h$ is small enough, $\left(z-B_{\theta}^{\natural}\right)^{-1}$ exists and is holomorphic in $z$. In particular it follows that

$$
\int \partial_{\bar{z}} \tilde{f}(z)\left(z-B_{\theta}^{\natural}\right)^{-1} d z \wedge d \bar{z}=0
$$

and since $\left(z-\widetilde{B}_{\theta}\right)^{-1}=\left(z-B_{\theta}^{\natural}\right)^{-1}+\left(z+\widetilde{B}_{\theta}\right)^{-1} \chi^{w}\left(x, h D_{x}\right)\left(z-B_{\theta}^{\natural}\right)^{-1}$,

$$
f\left(\widetilde{B}_{\theta}\right)=\frac{1}{2 \pi i} \int \partial_{\bar{z}} \tilde{f}(z)\left(z-\widetilde{B}_{\theta}\right)^{-1} \chi^{w}\left(x, h D_{x}\right)\left(z-B_{\theta}^{\natural}\right)^{-1} d z \wedge d \bar{z} .
$$

Since the trace class norm of $\chi^{w}\left(x, h D_{x}\right)$ is $\mathscr{O}\left(h^{-n}\right)$, the almost analyticity of $\tilde{f}$ yields the desired bound.

Let us recall our assumption (1.7),

$$
\left|\operatorname{tr}\left(g\left(P_{0}\right) \chi_{0}\right)\right| \leq C \Phi(C / h)
$$

for $g \in C_{0}^{\infty}(\mathbb{R})$ and $\chi_{0} \in C_{0}^{\infty}\left(\mathbb{R}^{n}\right)$. Combined with Lemma 7.2 and the comment concerning $f\left(\widehat{B}_{\theta}\right)$ preceding it we obtain

$$
M\left(R_{2}, h\right) \leq C \Phi\left(C / h_{1}\right),
$$


with " $C$ " denoting a new constant in every new formula. We now proceed to estimate the number of eigenvalues of $\left(P_{\theta}-\omega_{0}\right)^{1 / 3}-\omega_{1}$ in a disc $D\left(0, R_{1}\right)$. This is done by adapting the method used in $\S 4$ of [23].

Let the eigenvalues of $Z_{\theta}$ in $D\left(0, R_{1}\right)$ be $z_{1}, \ldots, z_{N},\left|z_{j}\right| \leq\left|z_{j+1}\right|$, listed according to their multiplicity. Let

$$
\mu_{1} \leq \mu_{2} \leq \cdots \leq \inf \sigma_{\text {ess }}\left(B_{\theta}^{1 / 2}\right)
$$

be an enumeration of all the discrete eigenvalues of $B_{\theta}^{1 / 2}$ below the essential spectrum and then possibly (if there are only finitely many eigenvalues) the infimum of the essential spectrum repeated infinitely many times. We recall the Weyl inequality (see [4] and, for this particular case, Appendix A of [23]):

$$
\mu_{1} \cdots \mu_{N} \leq\left|z_{1}\right| \cdots\left|z_{N}\right| \text {. }
$$

We also observe that, by Lemma 7.1, $\mu_{1} \geq r$ if $h$ is small and where $r$ is independent of $h$. Returning to (7.7) we want to prove that

$$
N \leq C\left(r, R_{1}, R_{2}\right) C \Phi(C / h) \text { if } M\left(R_{2}, h\right) \leq C \Phi(C / h) .
$$

Let us assume that $N \geq C \Phi(C / h)$, otherwise we can take $C\left(r, R_{1}, R_{2}\right)=1$. Then (7.7) gives

$$
r^{C \Phi(C / h)} R_{2}^{N-C \Phi(C / h)} \leq R_{1}^{N},
$$

since $\mu_{j}>R_{2}$ for $j>C \Phi(C / h)$ and $\mu_{1}>r$, while $\left|z_{j}\right|<R_{1}$. Thus

$$
\left(R_{2} / R_{1}\right)^{N} \leq\left(R_{2} / r\right)^{C \Phi(C / h)}
$$

so that

$$
N \leq C \frac{\log R_{2} / r}{\log R_{2} / R_{1}} \Phi(C / h)=\widetilde{C} \Phi(C / h) .
$$

Returning to the operator $P_{\theta}$, we see from Proposition 4.3 that the number of eigenvalues (with multiplicity) in $\Omega_{0}$ is equal to the number of eigenvalues of $\left(P_{\theta}-\omega_{0}\right)^{1 / 3}$ in $\Omega$ (see Figures 7.1 and 7.2), which in turn is less than the number of eigenvalues of $\left(P_{\theta}-\omega_{0}\right)^{1 / 3}-\omega_{1}$ in $D\left(0, R_{1}\right)$.

Thus the number $N_{\Omega}(h)$ of resonances of $P_{\theta}$ in $\Omega$ satisfies

$$
N_{\Omega}(h) \leq N \leq \widetilde{C} \Phi(C / h) .
$$

We now consider

$$
N(r)=\sharp\left\{\lambda:|\lambda| \leq r, \lambda \text { a pole of }\left(P-\lambda^{2}\right)^{-1}\right\}, \quad r \geq 1,
$$

where we include the poles according to their multiplicities. The estimate of $N(r)$ will easily follow from summing up the contributions from expanded annuli. For this we drop the semiclassical parameter $h$, and consider the number of scattering poles in

$$
N(R, h)=\left\{\lambda:-\pi / 2 \leq \arg \lambda \leq 0, R^{1 / 2} h^{-1} \leq|\lambda| \leq 2 R^{1 / 2} h^{-1}\right\} .
$$


We have $N(R, h)=N_{\Omega}(h)$, and hence the number of poles $\lambda$ with $L / 2 \leq$ $|k| \leq L$ is bounded by $C \Phi(C L)$ when $L \geq C$. For a fixed large $L_{0}$ choose $k \in \mathbb{N}$ such that $2^{-k} r \in\left[L_{0} / 2, L_{0}\right]$. Then

$$
N(r) \leq N\left(L_{0}\right)+C \sum_{j=0}^{k-1} \Phi\left(C 2^{-j} r\right) \leq N\left(L_{0}\right)+C \widetilde{C} \Phi(C r) \sum_{j=1}^{k-1} 2^{-\delta j} \leq \widehat{C} \Phi(C r),
$$

which is our final estimate.

Remark 7.1. We consider a flat torus $\mathbb{T}_{R}, R>R_{0}+a_{0}$, obtained by identifying the sides of $\left\{x \in \mathbb{R}^{n}:\left|x_{i}\right|<R, i=1, \ldots, n\right\}$, and let $P_{0}^{\sharp}$ be the unbounded selfadjoint operator on

$$
\mathscr{H}^{\sharp}=\mathscr{H}_{R_{0}} \oplus L^{2}\left(\mathbb{T}_{R} \backslash B\left(0, R_{0}\right)\right)
$$

defined by $P_{0}^{\sharp}=P_{0}(\chi u)-h^{2} \Delta(1-\chi) u$, where $\chi \in C_{0}^{\infty}\left(B\left(0, R_{0}+\varepsilon\right)\right)$ is equal to 1 near $\overline{B\left(0, R_{0}\right)}$. The domain $\mathscr{D}^{\sharp}$ of $P_{0}^{\sharp}$ is $\left\{u \in \mathscr{H}^{\sharp}: \chi u \in \mathscr{D},(1-\chi) u \in H^{2}\right\}$, and we define a corresponding scale of spaces $\mathscr{D}^{\sharp, \alpha}$ with $D^{\sharp, 1}=\mathscr{D}^{\sharp}$. As in $\S 6$, we define the seminegligible operators $(\mathrm{sn})$ and the semitrace negligible operators (stn). The proof of Proposition 6.1 implies that $\chi_{1}\left(z-P_{0}^{\sharp}\right)^{-1} \chi_{2}$ is (stn) if $\chi_{1}, \chi_{2} \in C^{\infty}\left(\mathbb{T}_{R}\right)$ are constant on $\overline{B\left(0, R_{0}\right)}$ and have disjoint supports. As in $\S 6$ we get that modulo stn operators

$$
\left(z-P_{0}^{\sharp}\right)^{-1} \chi \equiv \tilde{\chi}\left(z-P_{0}\right)^{-1} \chi
$$

when $\chi, \tilde{\chi} \in C_{0}^{\infty}\left(B\left(0, R_{0}+\varepsilon\right)\right), \chi=1$ near $\overline{B\left(0, R_{0}\right)}$, and $\tilde{\chi}=1$ near $\operatorname{supp} \chi$. Moreover,

$$
\left(z-P_{0}^{\sharp}\right)^{-1} \chi=(1-\psi)\left(z-\widehat{P}_{0}^{\sharp}\right)^{-1} \chi
$$

modulo stn operators if we now assume that $\chi \in C_{0}^{\infty}\left(\mathbb{T}_{R} \backslash \overline{B\left(0, R_{0}\right)}\right), \psi \in$ $C_{0}^{\infty}\left(\mathbb{T}_{R}\right)$ is equal to 1 near $\overline{B\left(0, R_{0}\right)}$, and $\operatorname{supp} \psi \cap \operatorname{supp} \phi=\varnothing$. Here $\widehat{P}_{0}^{\sharp}=$ $-h^{2} \Delta_{\mathbb{T}_{R}}$. Then (cf. Theorem 6.4) modulo trace negligible operators (with respect to the new scale of spaces):

$$
g\left(P_{0}^{\sharp}\right) \equiv \tilde{\chi}_{1} g\left(P_{0}\right) \chi_{1}+\left(1-\psi_{2}\right) g\left(\widehat{P}_{0}^{\sharp}\right) \chi_{2}
$$

with $\chi_{1}, \tilde{\chi}_{1} \in C_{0}^{\infty}\left(B\left(0, R_{0}+\varepsilon\right)\right), \tilde{\chi}_{1}=1$ near $\operatorname{supp} \chi_{1}, \chi_{2} \in C^{\infty}\left(\mathbb{T}_{R}\right), 1=$ $\chi_{1}+\chi_{2}, \psi_{2}=1$ near $\overline{B\left(0, R_{0}\right)}$, supp $\psi_{2} \cap \operatorname{supp} \chi_{2}=\varnothing$. Taking the traces of (7.8) we get for any $g \in C_{0}^{\infty}(\mathbb{R})$,

$$
\operatorname{tr} g\left(P_{0}\right) \chi_{1}=\operatorname{tr} g\left(P_{0}^{\sharp}\right)+\mathscr{O}\left(h^{-n}\right) .
$$

We shall now consider the $h$-independent operator $P^{\sharp}, P_{0}^{\sharp}=h^{2} P^{\sharp}$. We assume that the number of eigenvalues of $P^{\sharp}, \lambda$, with $|\lambda| \leq r^{2}$ is bounded by $C \Phi(C r)$ for $r \geq 1$. Then $\left|\operatorname{tr} g\left(P_{0}^{\sharp}\right)\right| \leq C \Phi(C / h)$ and (7.9) gives $\left|\operatorname{tr} g\left(P_{0}\right)\right| \leq$ 
$C \Phi(C / h)$. In other words, we get (1.7) from a corresponding assumption on the number of eigenvalues of $P^{\sharp}$ above.

\section{ACKNOWLEDGMENT}

The second author would like to thank Université de Paris-Sud, Orsay, for the hospitality in June 1990, at which time part of this project was carried out.

\section{Note ADDED IN PROOF}

G. Vodev has recently provided an alternative proof of Theorem 1.2. His method avoids complex scaling and is based on the techniques of [20, 26, 29] and the proof of Theorem 1.1 here. The methods developed in this paper, however, have been used by the authors to obtain more accurate bounds near the real axis.

\section{REFERENCES}

1. J. Aguilar and J. M. Combes, A class of analytic perturbations for one-body Schrödinger Hamiltonians, Comm. Math. Phys. 22 (1971), 269-279.

2. E. Balslev and J. M. Combes, Spectral properties of many-body Schrödinger operators with dilation analytic interactions, Comm. Math. Phys. 22 (1971), 280-294.

3. Y. Colin de Verdiere, Pseudo Laplacians. II, Ann. Inst. Fourier (Grenoble) 33 (1983), 89113.

4. I. Gohberg and M. Krein, Introduction to the theory of linear non-self-adjoint operators, Amer. Math. Soc., Providence, RI, 1969.

5. B. Helffer and A. Martinez, Comparison entre les diverses notions de résonances, Helv. Phys. Acta 60 (1987), 992-1003.

6. B. Helffer and J. Sjöstrand, Résonances en limite semi-classique, Bull. Soc. Math. France, mémoire no. 24/25 114 (1986).

7. __, Analyse semi-classique pour l'équation de Harper, Bull. Soc. Math. France 116 (1988).

8. __, Diamagnetism and de Haas-van Alphen effect, Ann. Inst. H. Poincaré. Phys. Théor. 52 (1990), 303-375.

9. L. Hörmander, The analysis of linear partial differential operators. I-IV, Springer-Verlag, Berlin-New York, 1983.

10. W. Hunziker, Distortion analyticity and molecular resonance curves, Ann. Inst. H. Poincaré. Phys. Théor. 45 (1986), 339-358.

11. A. Intissar, On the value distribution of the scattering poles, preprint.

12. __ A polynomial bound on the number of scattering poles for a potential in even dimensional space $\mathbb{R}^{n}$, Comm. Partial Differential Equations 11 (1986), 367-396.

13. P. Lax and R. Phillips, Scattering theory, Academic Press, New York, 1967.

14. G. Lebeau, Fonctions harmoniques et spectre singulier, Ann. Sci. École Norm. Sup. (4) 13 (1980), 269-291.

15. A. Martinez, Prolongement des solutions holomorphes de problèmes aux limits, Ann. Inst. Fourier (Grenoble) 35 (1) (1985), 93-116.

16. A. Melin, Trace distributions associated to the Schrödinger operator, University of Lund, preprint, 1990.

17. R. B. Melrose, Scattering theory and the trace of the wave group, J. Funct. Anal. 45 (1982), 29-40.

18. $\ldots$, Polynomial bounds on the number of scattering poles, J. Funct. Anal. 53 (1983), 287303. 
19. _ Growth estimates for the poles in potential scattering, unpublished, 1984.

20. __ Polynomial bounds on the distribution of poles in scattering by an obstacle, Journees "Equations aux Dérivées Partielle," Saint-Jean-de Montes, 1984.

21. _ Weyl asymptotics for the phase in obstacle scattering, Comm. Partial Differential Equations 13 (1988), 1431-1439.

22. W. Müller, Spectral geometry and scattering theory for certain complete surfaces of finite volume, preprint.

23. J. Sjöstrand, Geometric bounds on the density of resonances for semiclassial problems, Duke Math. J. 60 (1990), 1-57.

24. __ Estimates on the number of resonances for semi-classical Schrödinger operators, (Proc. VIII Latin American School of Mathematics, 1986) Lecture Notes in Math., vol. 1324, Springer-Verlag, Berlin-New York, 1988, pp. 286-292.

25. B. Vainberg, Asymptotic methods in equations of mathematical physics, Gordon and Breach, 1988.

26. G. Vodev, Sharp polynomial bounds on the number of scattering poles for metric perturbations of the Laplacian in $\mathbb{R}^{n}, n \geq 3$ odd, Math. Ann. (to appear).

27. M. Zworski, Distribution of poles for scattering on the real line, J. Funct. Anal. 73 (1987), 277-296.

28. _ Sharp polynomial bounds on the number of scattering poles of radial potentials, J. Funct. Anal. 82 (1989), 370-403.

29. $\ldots$ Sharp polynomial bounds on the number of scattering poles, Duke Math. J. 59 (1989), 311-323.

Départment de Mathématiques, Université de Paris-Sud, F-91405 Orsay, France AND URA 760, CNRS

Department of Mathematics, Harvard University, Cambridge, Massachusetts 02138 